\title{
1 Estimate of eddy energy 2 generation/dissipation rate in the world ocean 3 from altimetry data \\ 4
}

\author{
Manuscript for institutional repository WHOAS of the MBLWHOI Library
}

\section{8}

(1) 2 (1) (1) (1) (1)

\section{Chi Xu}

Key Laboratory of Tropical Marine Environmental Dynamics, South China Sea Institute of Oceanology, Chinese Academy of Sciences, Guangzhou, China, 510301

Graduate School of Chinese Academy of Sciences, Beijing 100049, China

\section{Xiao-Dong Shang (Corresponding author)}

Key Laboratory of Tropical Marine Environmental Dynamics, South China Sea Institute of Oceanology, Chinese Academy of Sciences, Guangzhou, China, 510301

e-mail: $\underline{x d s h a n g @ s c s i o . a c . c n}$

tel: $+86-20-89024731$

fax:+86-20-89024731

Rui Xin Huang

Woods Hole Oceanographic Institution, Woods Hole, MA 02543, USA

\section{Citation: Xu C., X.-D. Shang, and R.X. Huang, 2011: Estimate of eddy energy}

generation/dissipation rate in the world ocean from altimetry data. Ocean Dynamics, 61(4):525- 
2 Abstract

4 Assuming eddy kinetic energy is equally partitioned between the barotropic mode 5 and the first baroclinic mode and using the weekly TOPEX/ERS merged data for 6 the period of 1993 2007, the mean eddy kinetic energy and eddy available 7 gravitational potential energy in the world oceans are estimated at $0.157 \mathrm{EJ}$ and $80.224 \mathrm{EJ}$; the annual mean generation/dissipation rate of eddy kinetic energy and 9 available gravitational potential energy in the world oceans is estimated at 0.203 10 TW. Scaling and data analysis indicate that eddy available gravitational potential 11 energy and its generation/dissipation rate are larger than those of eddy kinetic 12 energy.

13 High rate of eddy energy generation/dissipation is primarily concentrated in eddy14 rich regions, such as the Antarctic Circumpolar Current and the western boundary 15 current extensions. Outside of these regimes of intense current, the energy 16 generation/dissipation rate is 2 to 4 orders of magnitude lower than the peak 17 values; however, along the eastern boundaries and in the region where 18 complicated topography and current interact the eddy energy 19 generation/dissipation rate is several times larger than those in background. 


\section{I Introduction}

Eddies are the most important component of the oceanic circulation. Scale analysis indicates that eddy kinetic energy (EKE) is two orders of magnitude

6 larger than the mean flow kinetic energy, and eddy available potential energy is

7 one order of magnitude larger than eddy kinetic energy (Gill et al. 1974; Huang

8 2010). Satellite altimetry data analysis indicates that at least on the sea surface of

9 the subtropical gyres, EKE is indeed about 100 times larger than the mean flow

10 kinetic energy; however, this ratio is reduced to approximately 10 in most part of

11 the Antarctic Circumpolar Current (ACC) (Wunsch 2007 Plate 6). EKE is most

12 contained in the form of geostrophic (or mesoscale) eddies on scales of 50 to 100

$13 \mathrm{~km}$ and time scale of 10 100 days; these eddies dominate the oceanic kinetic 14 energy at sub-inertial frequencies at mid- and high-latitudes (Ferrari and Wunsch

15 2009).However, it is clear that at this time we have no reliable theory and data for 16 eddy energy generation/dissipation rate in the world oceans. Since this is a

17 critically important component of the global energy budget, a clear dynamical

18 picture and a detailed balance are most desirable. Hence, we postulate a method to

19 combine altimetry data with a hydrographic climatology; this method can provide

20 useful information about the size of eddy-related energy reservoirs, including

21 potential and kinetic energy, in the world ocean, and the associated conversion 22 rates.

23 It has been long recognized that mesoscale eddies play important roles in the 24 energetics of the global oceans. In the 1970s, the first international field program 25 POLYMODE aimed at observing mesoscale eddies in the oceans was organized 26 (Gould et al. 1974). Despite grand technique challenges associated with observing 
1 eddies in the oceans, much progress was made. In particular, regimes of high values of EKE in the Gulf Stream Extension, the Kuroshio Extension and the ACC were identified (Wyrtki et al. 1976; Richardson 1983). With the advance of satellite altimetry in 1990s, nearly synoptical global pictures of the EKE distribution (Cheney et al. 1983; Zlotnicki et al. 1989; Shum et al. 1990; Stammer 1997; Ducet et al. 2000) are provided. With the improvement in remote sensing technique and accumulation of data, more precise pictures of the spatial structure and temporal evolution of the eddy field are immerging.

Most previous studies have been primarily focused on EKE, often calculated as half of the squared geostrophic velocity. In a stratified fluid, both the kinetic energy and available gravitational potential energy (AGPE) are important. Scaling indicates that most of the eddy energy may be stored in the form of eddy available gravitational potential energy (EAGPE), which is defined as the difference in gravitational potential energy between a reference state and the physical state associated with an eddy. However, this aspect of eddy energetics has not received much due attention.

As discussed in Feng et al. (2006), the AGPE is very sensitive to the choice of the reference state. For a person walking on a flat land, his AGPE seems rather small. However, when he sees a deep well by the road side, he realizes that his AGPE can be huge in comparing with the bottom of the well. Early studies of basin-scale AGPE by Oort et al. (1994) was based on a reference state obtained by horizontally averaging the global stratification. Such a formulation is, however, not suitable for the study of basin-scale circulation. A more suitable definition derived from the original definition of available potential energy should be used, and a computational algorithm including the compressibility of seawater and realistic topography was developed by Huang $(2005,2010)$. On the other hand, 
1 for the study of mesoscale eddies in the oceans, the locally averaged stratification

2 can be used as the reference state. Accordingly, the EAGPE in the world oceans 3 was estimated at $(1-8) \mathrm{EJ}\left(1 \mathrm{EJ}=10^{18} \mathrm{~J}\right.$, Feng et al. 2006). Furthermore, the global

4 distribution of EAGPE is closely linked to the strong density fronts and currents

5 in the oceans, implying that baroclinic instability could be the major mechanism

6 and energy source supporting these regimes of high EAGPE. In the present study,

7 we use a two-layer model to study the structure of an eddy; the reference state is

8 defined as the state with no free surface elevation caused by eddy. The appropriate

9 EAGPE algorithm can be derived from such a simple layer model.

10 Mesoscale eddies in the ocean evolve with time through the following

11 processes: eddy generation through baroclinic/barotropic instability or directly

12 forced by wind perturbations; energy transfer through eddy-eddy interaction and

13 eddy-mean flow interaction; and finally through many dynamic processes eddies

14 lose their energy and eventually die.

15 The generation of eddies in the oceans may be linked to both the atmospheric 16 forcing and the instability in the oceans. Frankignoul and Muller (1979)

17 postulated that mesoscale eddies were mainly forced by the fluctuating winds;

18 they put the energy source due to atmospheric forcing at $0.05 \mathrm{TW}$. Comparing this

19 level of energy source with other sources, wind fluctuations do not seem to play a

20 dominant role (Wyrtki et al. 1976; Stammer et al. 2001).

21 The other source of eddy has been identified as the instability in the oceans, 22 including both baroclinic and barotropic instability. These dynamical processes

23 have been studied extensively and summarized in textbooks, Pedlosky (1987).

24 Observations confirmed the claim of instability theory. For example, Stammer 25 (1997) found eddy variability was positively correlated with the mean horizontal 26 density gradients; thus, the internal instability is a primary source of eddy because 
1 large horizontal density gradient means strong baroclinic instability. Hydrographic

2 data analysis indicated that the ocean is baroclinically unstable everywhere (Smith

3 2007; Killworth and Blundell 2007), suggesting the source of eddy energy is

4 available in the oceans. Hence, the release of potential energy through baroclinic

5 instability can be a major mechanism sustaining the generation of mesoscale

6 eddies.

$7 \quad$ How much eddy energy is actually generated through baroclinic instability?

8 Using the commonly accepted Gent-McWilliams scheme, Huang and Wang (2003)

9 made an attempt of estimate the conversion rate from the mean-state gravitational

10 potential energy to eddy energy. Since eddy parameterization remains a crude

11 numerical technique, the conversion rate is rather sensitive to the choice of

12 parameter. A close examination was taken by Wunsch and Ferrari (2004), and they

13 put the estimate at 0.2 0.8 TW. In a more recent review, Ferrari and Wunsch

14 (2009) put this conservation rate at $0.3 \mathrm{TW}$.

15 Obviously, the conversion rate is limited by the rate of wind energy input to

16 the surface current; the estimate of this rate is $0.85 \sim 1 \mathrm{TW}$ according to the

17 studies by Wunsch (1998), Huang et al. (2006) and the most recent study by Scott

18 and $\mathrm{Xu}$ (2009). Thus, the eddy energy generation rate should be a fraction of 1

19 TW. However, due to the limitation of in-situ observations and computer power,

20 no reliable estimate of this rate has been published so far.

21 Another question is how eddies lose their energy. Due to the limitation of in-

22 situ observations and computer power, we have no clear dynamic picture for this

23 critically important component of the world ocean energetics.

24 Eddies may lose their energy through the following processes: bottom drag,

25 loss of balance (or called surface frontogenesis which results from eddy stirring

26 and implies an energy cascade from the first baroclinic mode to scales smaller 
1 than the first deformation radius), interactions with the internal wave field,

2 continental margin scattering/absorption and suppression by wind work. Some of

3 these processes were briefly discussed by Ferrari and Wunsch (2009); however,

4 most of these items remain unexplored.

5 Some observation data suggested that eddy dissipation is closely linked to

6 rough topography, e.g., current meter record study by $\mathrm{Fu}$ et al. (1982), altimeter

7 data analysis by Gille at al. (2000) and model study by Arbic and Flierl (2004).

8 Wunsch and Ferrari (2004) estimated this rate of energy loss at 0.4 TW. Using

9 moored current meter records and altimetry data, Sen et al. (2008) reexamined the

10 bottom drag and suggested that the global dissipation rate of low-frequency flow

11 by quadratic bottom boundary layer drag falls within the range of 0.2 to $0.8 \mathrm{TW}$.

12 Based on high-resolution global simulations, Arbic et al. (2009) put the global

13 dissipation rates by quadratic bottom boundary layer drag at 0.14 to 0.65 TW.

14 Although these studies seem to give a rather high upper limit for the rate of eddy

15 energy dissipation through bottom friction, it is questionable whether bottom

16 friction can take up such a large portion of the total eddy energy.

17 Although the eddy-related energy and conversion rates are critically

18 important, progress in diagnosis based on observation data has been rather slow.

19 Satellite altimetry data is the most powerful tool currently available in collecting

20 synoptic data of eddy-related sea surface height anomaly on global scale. In order

21 to incorporate the vertical structure of eddies, the simplest approach is to use a

22 two-layer model to infer the baroclinic structure of eddies. Thus, our study is

23 focused on the diagnosis of eddy energy generation/dissipation rates based on

24 reliable merged satellite altimetry data and an equivalent two-layer model.

In Section 2, we discuss eddy energy in the form of EKE and EAGPE. A 26 common approach is using the main pycnocline (thermocline) as the interface of a 
1 two-layer model. Following the analysis by Flierl (1978), an equivalent two-layer

2 model (EQ-model) is defined, based on the optimal parameter choice calculated

3 from the first mode of the continuously stratified model. In this way, eddy energy

4 is linked to the free surface elevation anomaly observed from satellite data. In

5 Section 3, the data processing and calculation algorithms are presented. The

6 results of our analysis are presented in Section 4, and we conclude in the last

$7 \quad$ Section 5.

8

92 Two-layer model and calculation algorithms

10 Mesoscale eddy energy consists of two parts: EKE and EAGPE. Eddies can

11 be classified as barotropic eddy, baroclinic eddy of mode 1, mode 2 and so on. In

12 theory, eddy energy calculation should include contribution from all possible

13 modes. However, such a calculation requires information about the vertical

14 structure of eddies, which is not available from satellite altimetry data only.

15 Wunsch (1997) went through a detailed analysis for all current meter data

16 available at that time, and his results indicated that most part of eddy kinetic

17 energy is contained in the first baroclinic mode. Forget and Wunsch (2007)

18 analyzed all hydrographic data in the global oceans and came to a similar

19 conclusion: "Over the global ocean, the interpretation of the SSH variability as the

20 vertical displacement signature of the first baroclinic mode is a reasonable

21 approximation." Ferrari and Wunsch (2010) noted that at periods beyond one day,

22 kinetic energy of a water column is roughly equally partitioned between the

23 barotropic mode and the first baroclinic mode. Thus, we will use this as a working

24 assumption. 
7 is presented in Appendix B, where the reason why this model is better than the 8 traditional two-layer model is presented.

\section{2.2. The calculation of the EKE and the EAGPE}

11 layer were computed from the SSHA as $u_{1}=-g \eta_{y} / f$ and $v_{1}=g \eta_{x} / f$. Assume

12 there are $n$ grid points within the closed sea surface height anomaly contour of an

13 eddy, the mean geostrophic velocity $\left\langle V_{1}\right\rangle$ in the upper layer of the eddy is

$14<V_{1}>=\sum_{i=1}^{n}\left(\sqrt{u_{1, i}{ }^{2}+v_{1, i}{ }^{2}}\right) / n$.

15 The total geostrophic kinetic energy of each eddy is

$16 \quad E K E=\sum_{i=1}^{n} 0.5\left(\nabla \eta_{i} / f\right)^{2} \bar{\rho} A_{i} H_{1, i} H_{i} / H_{2, i}$,

17 where $\bar{\rho} \simeq 1030 \mathrm{~kg} / \mathrm{m}^{3}$ is the reference density $H_{1, i}, H_{2, i}$ are the upper, lower

18 layer thickness and $H_{i}=H_{1, i}+H_{2, i}$ is the total thickness at grid $i$.

$20 \quad E A G P E=\sum_{i=1}^{n} \bar{\rho} g \eta_{i}^{2}\left(H_{i} / H_{2, i}\right)^{2} A_{i} / 2 \varepsilon_{i}$

21 Our discussion above is focused on the first baroclinic mode. In general, eddy

22 energy can exist in quite different forms. As discussed by Ferrari and Wunsch 23 (2010), eddy motions in the ocean can be described in terms of the Quasi- 
1 Geostrophic modes in the oceanic interior, plus the so-called Surface Quasi-

2 Geostrophic solutions (Lapeyre 2009). Since the traditional QG modes are defined

3 for the ocean at rest, it is may not be the best way to represent motions observed

4 in the ocean. On the other hand, the traditional QG modes are defined from the

5 unforced solutions of the homogeneous Sturm-Liouville system, and these modes

6 form an orthogonal and complete base; thus, any function has a unique (and

7 convergent) expression in this base. Hence, we can use these modes as the base

8 and assume that the eddy energy is partitioned as follows

$9 \quad E=c_{0} E_{b t}+c_{1} E_{b c, 1}+c_{2} E_{b c, 2}+\ldots$.

10 In this study our focus is on the first two terms only; accordingly, the SSHA

11 signals are separated into two parts

$12 \eta=\eta_{b t}+\eta_{b c}, \eta_{b t}=\alpha \eta, \eta_{b c}=(1-\alpha) \eta$

13 where $\eta_{b t}$ and $\eta_{b c}$ are the barotropic and baroclinic components, and $\alpha \in[0,1]$

14 is the fraction. To choose this fraction we assume that the total kinetic energy

15 partition can be written as

$16 k e=k e_{b t}+k e_{b c}, k e_{b t}=c \cdot k e, k e_{b c}=(1-c) k e$

17 For each grid, the vertically integrated kinetic energy is

$18 \quad k e_{b c}=0.5 \bar{\rho} A_{i}\left(\nabla \eta_{b c} / f\right)^{2} H_{1, i} H_{i} / H_{i, 2}$,

$19 k e_{b t}=0.5 \bar{\rho} A_{i}\left(\nabla \eta_{b t} / f\right)^{2} H_{i}$.

20 From Eqs. $(5,6,7,8)$, we obtain

$21 \quad \alpha=\left(1+\sqrt{\frac{H_{2, i}}{H_{1, i}} \frac{1-c}{c}}\right)^{-1}$,

$22 \quad E K E=\sum_{i=1}^{n}(1-\alpha)^{2} \bar{\rho} A_{i}\left(\nabla \eta_{i} / f\right)^{2} H_{1, i} H_{i} / H_{2, i}$ 
The available gravitational potential energy associated with the barotropic mode is 2 much smaller than the corresponding kinetic energy, thus can be omitted; the 3 available gravitational potential energy for an eddy is

$4 \quad E A G P E=\sum_{i=1}^{n}(1-\alpha)^{2} \bar{\rho} g \eta_{i}^{2}\left(H_{i} / H_{2, i}\right)^{2} A_{i} / 2 \varepsilon_{i}$.

5 On the sea surface, the percentage of the kinetic energy associated with the

6 first baroclinic mode and the barotropic mode is

$7 \quad R_{b c}=(1-\alpha)^{2} /\left[\alpha^{2}+(1-\alpha)^{2}\right]$

8 It is clear that c may be a function of space and time; however, as a first step in

9 reveal the eddy energetics, we will assume $c=0.5$ is a global constant, i.e., the 10 water-column integrated kinetic energy is equally-partitioned between the

11 barotropic mode and the first baroclinic mode; thus, we have

$12 \quad R_{b c}=H_{2} / H$

13 Thus, the surface kinetic energy is mostly associated with the first baroclinic

14 mode, as discussed by Wunsch (1997). In the following discussion, we will

15 present results based on the case with $c=0.5$, unless stated otherwise.

163 Data analysis

$17 \quad$ 3.1. The data

18 The weekly TOPEX/ERS merged data over period $1993 \sim 2007$ were used in

19 our analysis. We used the data covers the latitude band from $60^{\circ} \mathrm{S}$ to $60^{\circ} \mathrm{N}$ with a

20 horizontal average resolution of $0.333^{\circ} \times 0.265^{\circ}$. Since errors of altimeter data are

21 larger near the boundary, the sea surface height anomaly (SSHA) data over

22 regimes with depth shallower than 200 meters are abandoned. Many issues related

23 to the quality and utility of this data set have been discussed in previous studies, 
e.g. Chelton et al. (2007).

The stratification data is obtained from the WOA01 annual mean climatology of temperature and salinity. The vertical structure of $\mathrm{T}$ and $\mathrm{S}$ profiles at each $1^{\circ} \times$ $1^{\circ}$ grid point is linearly interpolated to vertically uniform grid of 50 meter interval. The squared buoyancy frequency $N^{2}(z)$ at each grid is calculated by the standard

Matlab subroutine: Seawater (provided by CSIRO MatLAB Seawater Library, Phil Morgan, CMR).

\subsection{Identifying and tracking mesoscale eddies}

Eddy-like character of variability (time scales of 100 day and space scales of $100 \mathrm{~km}$ ) can be identified from SSHA as follows. First, the SSHA fields were zonally high-pass filtered to remove large-scale heating and cooling effects (Chelton and Schlax, 1996). The resulting anomaly fields were high-pass filtered with filter cutoffs of $6^{\circ} \times 6^{\circ}$ to reduce mapping errors. The reasons of choosing high-pass filter are two folds. In general, the size of an eddy is smaller than $6^{\circ} \times 6^{\circ}$, especially at high latitudes. In addition, at lower latitudes perturbations are primarily in the form of linear Rossby waves with relatively large spatial scale; thus, with high-pass filtering applied to remove the large-scale SSHA, not much eddy signals are retained (Chelton et al. 2006).

Two criteria applied to identify eddies. 1) A closed contour of SSHA $= \pm 5 \mathrm{~cm}$; 2) the zonal and longitudinal spread of the area enclosed by SSHA contour are both at least $0.5^{\circ}$. The central location of the eddy is defined as the centroid of area within the closed SSHA contour. Since $f$ approaches zero near the equator, the eddy calculation is limited to $5^{\circ}$ off the equator.

Eddies are tracked from SSHA fields at consequent time steps as follows. If an eddy center at next step is located within a circle centralized at the center of an eddy at the previous time step, these two eddies are considered as the same eddy 
1 at these two time steps. To avoid jumping from one track to another, the radius of

2 the circle is restricted to $1^{\circ}$ of latitude.

3 Comparing eddy characteristics in our analysis with results from Chelton et al.

4 (2007) showed a good agreement in almost all important aspects, including the

5 global distribution of eddies, and eddy propagation velocities and direction. The

6 number of eddies in our results are slightly larger due to the high-pass filtering

7 enhances the eddy variability at higher spatial resolutions.

8 Analyzing the merged altimetry product over the 15 year data, approximately

9275,000 eddies were identified and the number of long-lived cyclonic

10 (anticyclonic) eddies with lifetime $\geq 4$ weeks were 51719 (51557); thus, $37.55 \%$

11 of the observed eddies were long-lived. The trajectories, the number per $1^{\circ}$ square

12 of long-lived eddies and their mean EKE (per unit mass) derived from the 13 geostrophic velocities are shown in Fig. 1.

14 Eddies are mainly concentrated in the vicinity of the major current systems.

15 At low latitudes (especially the equatorial band), at high latitudes, and in the 16 eastern basins eddy activity is much lower. Lack of eddies in these regimes may

17 be due to the fact that large-scale ocean waves there may dominate the observed

18 SSHA (Chelton et al. 2006). Eddies in the tropics propagate mostly westward;

19 while eddies in the western boundary current extensions have eastward velocity

20 components, which may be induced by the mean flow. Eddies in the ACC band

21 primarily propagate eastward due to the intense eastward current and the Westerly

22 wind. The mean EKE (per unit mass) shown in low panels of Fig. 1 is directly

23 calculated from the SSHA data. The pattern and strength of the EKE are in

24 excellent agreement with Stammer (1997, his Fig. 2) and Ducet et al. (2000, their

25 Plate 8). In conclusion, thus, the distribution and directly-derived energetics of

26 mesoscale eddies are very similar with results in previous publications. 
1 3.3. Calculation of the annual mean generation/dissipation rate of mesoscale

2 eddies

Through eddy identification and tracking, the time series of position and energy for an eddy were obtained and the total energy of an eddy at each moment in its lifetime was calculated as summation of EKE and EAGPE.

Assume that we have a time series of an eddy, including its position and the

7 SSHA at uniform time step of one week. In order to analyze the life cycle of the

8 eddy, we extrapolate this life of eddy to define the beginning and end of the eddy.

9 Eddy energy was first calculated in non-uniform grid points, and it was converted 10 into a $1^{\circ} \times 1^{\circ}$ grid data set. The 15 -year mean of sources/sinks at those grid points

11 is thus computed (see detail in Appendix C).

\section{Results}

\section{4.1. The interfacial depth}

14 The interfacial depth for the EQ-model can be determined by solving the 15 eigen value problem and inferred from Eq. (B3) in Appendix B, Fig. 2a. In 16 addition, this depth field is subjected to a constraint of $H_{1} \leq H / 2$ and a 17 smoothing.

18 Alternatively, the corresponding interfacial depth of the TH-model can be 19 diagnosed from climatological data. After the approximate range is set, the level 20 of maximum vertical temperature gradient in each station is diagnosed. For 21 stations with no subsurface temperature gradient maximum, the corresponding 22 depth is determined by interpolation from adjacent stations. The map after 23 smoothing is shown in Fig. 2 b.

24 At middle and low latitudes, these two maps share similar features. For 25 example, the equivalent interface depth in the Atlantic is slightly larger than that 26 in the Pacific Ocean and Indian Ocean. However, they are quite different at high 
1 latitudes. The upper layer thickness of the EQ-model is mostly deeper than 500 meters poleward of $40^{\circ}$. Within the central latitude band of ACC, especially south of $45^{\circ} \mathrm{S}$, the equivalent interface depth is on the order of $1000 \mathrm{~m}$. In comparison,

4 the thermocline depth of the TH-model is quite shallow at high latitudes, on the 5 order of 100-200 $\mathrm{m}$ only. In fact, the main thermocline outcrops along the

6 poleward edge of the subtropical gyre. Thus, at latitudes higher than the poleward

7 boundary of the subtropical gyres, there is no main thermocline or pycnocline. As

8 a result, it is rather difficult to define such an interface, and a dynamical meaning

9 of the TH-model seems unclear.

A close examination also reveals some difference exists at lower latitudes

11 between these two models. For example, within $20^{\circ}$ of the equator in the Pacific

12 Ocean, the equivalent interfacial depth of the EQ-model is approximately $200 \mathrm{~m}$,

13 but it rises to 400 meters in the east. On the other hand, the corresponding

14 interfacial depth in the TH-model is deep in the western equatorial Pacific Ocean

$15(150 \mathrm{~m})$, but it is shallow in the eastern equatorial Pacific Ocean (less than 100m).

16 However, the difference in the equatorial band does not really affect our

17 calculation in this study because the equatorial band turns out to be a zone of low 18 eddy activity within our approach, as discussed above.

19 The density step $\varepsilon$ for the EQ-model is calculated from Eq. (B4), upper panel

20 of Fig. 3. In compassion, the corresponding value for the TH-model is defined 21 as: $\mathcal{E}^{\prime}=\left(\overline{\rho_{\theta, \text { lower }}}-\overline{\rho_{\theta, \text { upper }}}\right) / \bar{\rho}$, where $\overline{\rho_{\theta, \text { lower }}}$ and $\overline{\rho_{\theta, \text { upper }}}$ are the mean potential 22 density for the layers above and below the main thermocline at each station. The 23 high density step near the Warm Water Pool in the equatorial Pacific reflects the 24 fact that stratification is very strong due to the warm and relatively fresh water 25 there. In comparison with the TH-model, density step obtained from the EQ26 model is relatively large in the core of ACC. According to Eq. (A18), this 
1 difference should give rise to a relatively low level of EAGPE. On the other hand,

2 the equivalent interfacial depth of the EQ-model is much deeper in this area.

3 According to Eq. (A17), this should give rise to a much higher EKE there. The

4 difference in these two models will be discussed further shortly. However, in the

5 following analysis, we will use the equivalent interfacial depth and the density

6 step inferred from the EQ-model, unless specifically stated otherwise.

\section{4.2. The total EKE and EAGPE}

We begin with the diagnosing AGPE and EKE from satellite data. The meridional distributions of zonally integrated EKE/EAGPE are shown in Fig. 4.

10 Eddy activity in the equatorial band is a low, as shown in previous studies. In the

11 Northern Hemisphere, high density of EKE and EAGPE appears around the

12 latitude band of $40^{\circ} \mathrm{N}$, which is closely related to the Gulf Stream and Kuroshio

13 recirculation. In the Southern Hemisphere, there are two peaks. The northern peak 14 around $40^{\circ} \mathrm{S}$ is related to the strong recirculation of the subtropical gyres, 15 especially the Agulhas Return Current in the South Indian Ocean, and the 16 confluence regimes of the subtropical gyre and the ACC. The second peak appears 17 around $50^{\circ} \mathrm{S}$, which is closely related to the strong eddy activity in connection 18 with the core of the ACC.

19 In addition, the distribution of zonally mean eddy lifetime of eddies is shown

20 in Fig. 4c. There are two peaks of eddy lifetime in each hemisphere, and the 21 global mean lifetime is about 4 weeks. Eddy lifetime gradually declines toward 22 the equator. The reason of the eddy lifetime distribution remains unclear.

23 Both the ratio of EAGPE over EKE and the ratio of eddy scale over the 24 radius of deformation vary greatly with latitude, Fig. 4d. At lower latitudes, 25 deformation radius is much larger than the mean eddy scale, and the energy ratio 26 is smaller than one near the equator. At high latitudes, deformation radius is much 
1 smaller than the mean eddy scale, while the energy ratio is increased to 2 or even

2 3. This is consistent with the explanation and Eq. (A19) in the Appendix A, which implies the ratio of baroclinic EAGPE over baroclinic EKE is equal to the squared ratio of the eddy scale to the radius of deformation. Since according to Eq. (6), we

5 have $k e_{b c}=0.5 k e$ and we omit the barotropic EAGPE in our calculation as

6 mentioned in Section 2.2, the ratio of baroclinic EAGPE over baroclinic EKE,

7 indicated by the thin dashed line, is theoretically twice the ratio of EAGPE over

8 EKE, depicted by the thin solid line. However, in Fig. 4d, on one hand, the

9 squared ratio of the eddy scale to the radius of deformation (not shown) is lower

10 than double the ratio of EAGPE over EKE, suggesting that the eddy scale

11 resolved from the SSHA fields may be underestimated. On the other hand, at mid-

12 latitudes around $25^{\circ}$ the energy ratio is larger than one while the radius ratio is

13 smaller than one; we have not yet found any plausible explanation, and thus this is

14 left for further study.

15 As shown in Fig. 5, regions of low ratio (no more than 2) are located within

16 the subtropical gyre, including their western boundary and extensions where

17 intense currents and eddy activity are quite strong. However, at high latitudes,

18 especially in the east part of the North and South Pacific Ocean, the ratio is quite

19 large where eddy generation is less active. The maximum of ratio is larger than 10.

20 At $45^{\circ} \mathrm{S}$ band, although the Rossby deformation radius is nearly the same, this

21 ration is quite large in the eastern part of the South Pacific and its conjunction

22 with ACC, indicating that the spatial scale of eddies there is much larger than the

23 deformation radius.

24 The total EKE/EAGPE diagnosed from the EQ-model is summarized and 25 compared with previous estimates, Tables 1 and 2. The total EKE in cyclones 26 (0.081 EJ) is slightly larger than that in anticyclones $(0.076 \mathrm{EJ})$; similarly, the 
1 total EAGPE in cyclone $(0.113 \mathrm{EJ})$ is slightly larger than that of anticyclones $2(0.111 \mathrm{EJ})$

The total EKE and EAGPE is $0.157 \mathrm{EJ}$ and 0.224 EJ, respectively, Table 1.

4 These values are much smaller than those reported in previous studies, Table 2.

5 For example, using data for the monthly mean velocity for the period from 1958

6 to 2001 taken from Simple Ocean Data Assimilation (SODA) data (Carton and

7 Giese, 2008), the mean kinetic energy of the world ocean is estimated at $1.46 \mathrm{EJ}$

8 (Huang 2010). Since most kinetic energy is in forms of eddy, this number can be

9 used as an estimate of EKE. Ferrari and Wunsch (2009) put the estimate as 2.6 EJ,

10 without giving the detail of their estimate. The large difference between EKE

11 diagnosed in the present study is about 10 times smaller than the values diagnosed

12 from numerical model of data assimilation in the SODA data.

13 The total EAGPE in the world oceans remains unclear. Early estimate, such

14 as Oort et al. (1994), of AGPE in the world oceans was based on dynamical

15 framework of mesoscale dynamics. As discussed by Huang (2010), using such a

16 formulation is, however, not suitable for the study of basin-scale circulation. A

17 more accurate formulation gave the estimate of AGPE at 1880 EJ (Huang 2005).

18 However, the contribution due to the available internal energy is negative, and the

19 algebraic sum of these two terms is 810 EJ. As discussed above, a suitable choice

20 of referenced state is of critical importance in calculating the AGPE. For the study

21 of eddy energetics, a reference state obtained by averaging the stratification within

22 a horizontal domain on the order of the first deformation radius is a good choice,

23 The total amount of EAGPE in the world oceans sensitively depends on the

24 choice of the reference state. Using either a $1^{\circ} \times 1^{\circ}$ or $2^{\circ} \times 2^{\circ}$ gird, the total

25 amount of EAGPE in the world ocean was estimated at 1-8 EJ (Feng et al. 2006).

26 These numbers are larger than the value of $0.224 \mathrm{EJ}$ obtained in this study. 
Thus, it is clear both the EKE and EAGPE estimates obtained in this study is much smaller than estimates obtained from theory and numerical models. In particular, EKE is one order of magnitude smaller than that obtained from numerical simulations. The large difference between our estimates and those from theory and numerical models may be due to the rather low spatial and horizontal resolutions used in collecting satellite data and the smoothing used in merging and analyzing the satellite data.

\subsection{The mean generation/dissipation rate of mesoscale eddies}

The generation/dissipation of mesoscale eddies is a key component of the general circulation because eddies take energy from the large-scale mean state through barotropic and baroclinic instability. Eventually, eddies dissipate their energy through many dynamical processes. However, most of these processes remain unclear at present time, and these processes are simply treated as either a net growth or a net dissipation of an eddy between two stations which the eddy occupied during the consequent time at two consequent time steps.

For eddies with lifetime $\geq 2$ weeks, the annual mean generation/dissipation rate of eddy energy was calculated based on the Eqs. $(10,11)$, Fig. 6. At the resolution available from satellite data, the spatial distribution of the generation and dissipation rate is practically the same. In addition, the maps for cyclonic eddies and anticyclonic eddies are quite similar, and the minor difference can be seen only in the zonal integrated distribution shown in Fig. 7. Thus, only maps of cyclonic eddy energy generation rate are presented here.

Comparing Fig. 6 with Fig. 1 indicates that eddies are abundant in the Kuroshio Extension, the Gulf Stream Extension and the ACC. In the Northern Hemisphere high eddy activity appears in the West Boundary Current (WBC) extensions, which seems directly linked to the instability of mean flow, with a 
1 energy generation rate on the order of $15 \mathrm{~mW} / \mathrm{m}^{2}$. The high energy generation rate regime in the North Pacific Ocean appears as a zonal band, $30^{\circ} \mathrm{N} \sim 42^{\circ} \mathrm{N}$, but it

3 extends further northeastward in the North Atlantic Ocean. In fact, one of the

4 highest rate areas is located as far as $50^{\circ} \mathrm{N}$. This northeastward extension of high energy generation rate seems directly linked to the strong eddy activity associated with the North Atlantic Current.

In the Southern Hemisphere, the high energy generation rate appears in the Brazil current, the Brazil-Malvinas Confluence (the recirculation in the Argentine basin), and the Agulhas Current and its retroflection. The corresponding currents and associated recirculation systems in the South Pacific Ocean does not appear as a regime of strong eddy generation. The equatorial band and a vast regime in the east part of the North and South Pacific Ocean turn out to be a zone of very low eddy generation. Some of these low eddy generation locations, such as the equatorial band, may be partially due to the data processing standards used in our analysis. There is another band of high energy generation rate in the South Hemisphere, closely associated with the core of ACC, Fig. 6.

In addition, eddy energy generation rate along the eastern boundary of the Pacific and off the western coast of Australia is one order of magnitude higher than the corresponding value in the adjacent interior ocean, suggesting local wave-induced generation mechanism of eddy (Zamudio et al. 2007) and eddy may generate or dissipate their energy at continental margin via relatively weak baroclinic instability, eddy-eddy interaction or eddy-wave interaction.

The characters of the zonally/meridionally integrated mean generation rates of eddies are shown in Fig. 7. In the meridional direction, there are three latitudinal bands of strong eddy energy generation. The first band is located around $35-40^{\circ} \mathrm{N}$, apparently linked to the recirculation of Gulf Stream and 
1 Kuroshio. The additional secondary peak around $50^{\circ} \mathrm{N}$ reflects the contribution

2 due to the North Atlantic Current. The local maximum energy generation rate of

$33.1 \mathrm{GW} /$ degree for cyclonic eddies locates at $38^{\circ} \mathrm{N}$, and the maximum rate of 3.0

$4 \mathrm{GW} /$ degree for anticyclonic eddies locates at $40^{\circ} \mathrm{N}$. This slight difference in

5 latitude band is because of the polarity of meanders of the Gulf Stream and

6 Kuroshio Extension, which are cyclonic on the south side of the flow and

7 anticyclonic on the north side of the flow.

8 The generation rate in the equatorial band is extremely low. South of $20^{\circ} \mathrm{S}$,

9 eddy energy generation rate gradually increases and reaches a large amplitude

10 around two bands of peak value, one close to $38^{\circ} \mathrm{S}$ and another one between $48^{\circ} \mathrm{S}$

11 and $55^{\circ} \mathrm{S}$.

12 From Fig. 7, it is clear that more than half of eddy energy is generated in the

13 Southern Hemisphere, especially near ACC. The energy generation rate at the core

14 of ACC reaches a value of $3.30 \mathrm{GW} /$ degree for cyclonic eddies and 3.16

$15 \mathrm{GW} /$ degree for anticyclonic eddies at $48^{\circ} \mathrm{S} \sim 56^{\circ} \mathrm{S}$. At $156^{\circ} \mathrm{E}$, contributions from

16 the Kuroshio Extension and the confluence east of Australia give rise to a high

17 peak of $1.18 \mathrm{GW} /$ degree for cyclonic eddies and $1.11 \mathrm{GW} /$ degree for anticyclonic

18 eddies, lower panels of Fig. 7. The largest peak at $48^{\circ} \mathrm{W}$ the highest peak (1.46

$19 \mathrm{GW} /$ degree for cyclonic eddies and $1.38 \mathrm{GW} /$ degree for anticyclonic eddies) is

20 due to the contributions from the Gulf Stream Extensions and the Brazil-Malvinas

21 Confluence in the Argentine Basin. The smaller peaks at longitudes of $0^{\circ} \sim 100^{\circ} \mathrm{E}$

22 result from the enhanced variability of eddy energy in the Agulhas Return Current

23 and the ACC band.

24 It is clear that we must pay close attention to the link between local energy

25 generation/dissipation and flow field and its interaction with topography. For

26 example, the Luzon Strait is a narrow gap between the South China Sea and the 
1 Northwest Pacific. Wang et al. (2003) found that west of the Luzon Strait eddies

2 are abundant. Our analysis indicates that this is a regime of relatively high eddy energy generation/dissipation rate. East of the Luzon Strait, Kuroshio brings in

4 fast current and strong shear. Strong eddy activity may be induced by the invasion

5 of Kuroshio (Yuan et al. 2006). Moreover, the rough topography in the strait and

6 the intense internal tide from the Pacific may also play a role in enhancing the 7 eddy activity.

8 Likewise, the intense currents and the rough bottom topography interact in

9 the Yucatán Channel through which the Gulf Stream flows from the Caribbean

10 Sea to the Gulf of Mexico, in the area that the ACC flows between the Falkland

11 Islands and South Georgia and the South Sandwich Islands, and in the eastside of

12 Australia where the East Australia Current exists, etc. The instability of mean flow

13 results from the flow-topography interaction may enhance the local eddy

14 generation rate. The annual mean energy conversion rates in these regions are $2 \sim$

156 times larger than those in the background (Figures not shown).

16 The total energy generation rate for mesoscale eddies with lifetime $\geq 2$ weeks

17 are listed in Table 3. The total generation/dissipation rate of mesoscale eddies is

$18 \quad 0.203 \mathrm{TW}$, and the rate for cyclonic eddies is lower than those of anticyclonic

19 eddies. It is important to notice that nearly half of eddy energy is generated in

20 ACC. The eddy energy generated in the Northern Hemisphere is much lower than

21 in the Southern Hemisphere.

22 As discussed above, eddy energy consists of two parts, EKE and EAGPE;

23 both components take part in the energy transfer and conversion. According to the

24 theory of baroclinic instability, at the horizontal scale of deformation radius, eddy

25 energy is approximately equally partitioned between these two components.

26 However, mesoscale eddies in the oceans gradually transfer their energy toward 
1 larger scale through eddy-eddy interaction. As scaling Eq. (A19) revealed, at scale

2 larger than the deformation radius, EAGPE is larger than EKE.

In many earlier studies the squared geostrophic velocity multiplied by a

4 factor of 0.5 is treated as the EKE, without including the contribution of layer

5 thickness. Furthermore, the EAGPE was seldom discussed. In view of the

6 importance of eddy energy partition, we went through the calculation and

7 separated these two components. In addition, the EKE component now contains

8 the contribution due to the mass of each eddy. Hence, the generation rates of EKE

9 and EAGPE for global cyclonic and anticyclonic eddies with lifetime $\geq 2$ weeks

10 are calculated (Fig. 8 top panel) and the global sums of these items are obtained

11 (Table 4). Accordingly, for the EQ-model the EKE generation rate of cyclonic

12 eddies is slightly lower than that of anticyclonic eddies. However, the EAGPE

13 generation rate of cyclonic eddies is slightly higher than that of anticyclonic

14 eddies. Further, like the ratio of EAGPE/EKE in Fig. 5, the ratio of cyclonic

15 EAGPE generation rates over EKE generation rates is shown in Fig. 8 lower panel.

16 For the global sums, in the EQ-model the EAGPE generation rate is about 1.3

17 times larger than that of EKE (Table 4).

18 As a comparison, we also include the results diagnosed from the TH-model

19 (See it in Appendix D). We believe that the results obtained from the EQ-model

20 are more reliable, and thus our discussion in this paper is based on this model.

\section{Summary and conclusion}

22 By assuming the barotropic and first baroclinic modes have equal kinetic 23 energy, the mean EKE and EAGPE are estimated at $0.157 \mathrm{EJ}$ and $0.224 \mathrm{EJ}$, and 24 the mean generation/dissipation rate of mesoscale eddies is estimated at $0.2 \mathrm{TW}$. 25 Previous estimates of the eddy generation and dissipation rate, such as Huang and 
1 Wang (2003) were based on rather crude eddy parameterization scheme. Due to

2 the highly uncertainty of the parameters used in their estimation, the accurate

3 value of this conversion remains unclear. In the latest review by Ferrari and

4 Wunsch (2009), a value of 0.3 TW was assigned, but no details were available. To

5 the best of our knowledge, no reliable estimate of eddy energy conversion rate

6 obtained from numerical model has been reported. Thus, the value of 0.2 TW may

7 be used as a target value.

The estimates of EKE and EAGPE reported in this study are much smaller

9 than those obtained from theory and numerical simulations. In particular, EKE is

10 at least 10 times smaller than the values based on theory and numerical simulation.

11 Such major gaps are primarily due to the rather low spatial and temporal

12 resolution of the altimetry data used in this study. Although the satellite altimetry

13 data we used has a horizontal grid resolution of 0.333 deg by $0.265 \mathrm{deg}$, features

14 can be resolved by this altimeter dataset are much coarser than this nominal

15 resolution. As a reviewer pointed out that the large difference between our

16 estimates and estimates based on theory and numerical simulations indicate that

17 there are a lot of mesoscale and submesoscale eddies which were not resolved by

18 this altimeter dataset. These smaller eddies may be responsible for a significant

19 amount of eddy kinetic and available potential energy, which are not included in

20 our estimates. Therefore, the EKE and EAGPE estimates in this study, as well as

21 their generation/dissipation rates, should be interpreted as the lower bounds for

22 the corresponding values. More accurate estimates for these important quantifies

23 are clearly needed for further study. It is clear that revealing the important role of

24 eddies in the ocean remains a grand challenge for observation technology, theory

25 and numerical simulation.

26 Despite the large gaps between the estimates from this study and those based 
1 on theory and numerical simulations, many aspects of our results may be useful

2 for understanding the role of eddies in the oceanic general circulation, such as the

3 spatial patterns of the EKE and EAGPE distribution, the patterns of the

4 generation/dissipation rate.

5 Although, a few estimates of eddy-related energy and conversion rates were

6 reported in the literature, but they were poorly constrained, and not always

7 consistent between each others. In particular, there were no estimates of EAGPE

8 based on satellite observations. We postulated a theoretical framework of the

9 calculation of EAGPE based on satellite SSHA observations. Thus, we believe

10 that our estimates set a set of consistent lower bounds for the eddy energetics in

11 the world oceans based on satellite observations.

12 One of the major uncertainties in our analysis is the working assumption that

13 EKE is equally participated between the barotropic mode and the first baroclinic

14 mode. (If we assumed that all kinetic energy is in the form of the first baroclinic

15 mode, the corresponding total eddy energy and its generation rate is estimated at

$160.646 \mathrm{EJ}$ and $0.345 \mathrm{TW}$ respectively, details of this analysis is not included).

17 Although Wunsch (1997) and Forget and Wunsch (2007) suggested that most

18 eddy energy on the sea surface is contained in the first baroclinic mode is a

19 reasonable approximation, recent studies raised some questions about this

20 assumption. For example, Lapeyre (2009) suggested that the SSHA signals may

21 be dominated by the surface geostrophic solutions. However, the surface

22 geostrophic solutions are surface trapped, and the corresponding interfacial

23 displacement in the deep part of the ocean is quite small; thus, such surface

24 trapped motions cannot be associated with a large amount of available potential

25 energy. Accordingly, the rate of eddy energy generation and dissipation would be

26 greatly reduced, and this may give rise to a completely different global energy 
1 balance. To resolve this critically important issue, further studies involving

2 analyzing in-situ observations or eddy-resolving numerical model output are

3 necessary.

There is a great uncertainty associated with the choice of filtering scale

5 because results obtained from processing satellite data are sensitive to the choice

6 of filtering scale. Different filtering scales may give quite different results. We

7 have carried out similar calculation using filtering scales from $5^{\circ} \times 5^{\circ}$ to $7^{\circ} \times 7^{\circ}$,

8 and the obtained eddy energy varies within the range of $0.196 \sim 0.577 \mathrm{EJ}$ and

9 energy generation rate varies within the range of $0.11 \sim 0.29 \mathrm{TW}$ (Table 1 , Table 3

10 and Table 4).

11 Our results suggest that most of eddy energy dissipation takes place in the 12 middle of the wind-driven circulation, especially the recirculation regimes and the

13 ACC. The regimes of strong dissipation in the Northern Hemisphere do not seem

14 to be directly linked to the bottom topography. Thus, energy dissipation through

15 interaction with bottom topography may not be the only way to dissipate eddy

16 energy. Other mechanisms, such as dissipation through loss of balance and

17 interacting with the atmosphere may play some kind of role.

19 Acknowledgement This study used altimeter data available by the AVISO Altimetry Operations 20 Center, plus hydrographic data World Ocean Atlas 2001 provided by the U.S. National 21 Oceanographic Data Center. This study is supported by Grants KZCX1-YW-12-01, 40976010, 2240776008.

\section{References}

Arbic, B. K., and G. R. Flierl (2004) Baroclinically unstable geostrophic turbulence in the limits of strong and weak bottom Ekman friction: Application to midocean eddies. J Phys Oceanogr 34:2257-2273. doi: 10.1175/1520-0485(2004)034<2257:BUGTIT>2.0.CO;2.

Arbic, B. K., J. F. Shriver, P. J. Hogan, H. E. Hurlburt, J. L. McClean, E. J. Metzger, R. B. Scott, A. Sen, O. M. Smedstad, and A. J. Wallcraft (2009) Estimates of bottom flows and bottom boundary layer dissipation of the oceanic general circulation from global high-resolution models. J Geophys Res 114: C02024. doi:10.1029/2008JC005072.

Carton, J. A., and Giese, B. S. (2008) A reanalysis of ocean climate using Simple Ocean Data 
Assimilation (SODA). Month Wea Rev 136:2999-3017. doi:10.1175/2007MWR1978.1.

Chelton, D. B., and M. G. Schlax (1996) Global observations of oceanic Rossby waves. Science 272:234-238. doi: 10.1126/science.272.5259.234.

Chelton, D. B., M. G. Schlax, R. M. Samelson, and R. A. de Szoeke (2007) Global observations of large oceanic eddies. Geophys Res Lett 34:L15606. doi:10.1029/2007GL030812..

Chelton, D. B., M. G. Schlax, R. M. Samelson, and R. A. de Szoeke (2007) Global observations of westward energy propagation in the ocean: Rossby waves or nonlinear eddies? Fall Meet. Suppl. abstract \#OS13E-07, AGU Fall Meeting, 87(52), San Francisco, CA, USA, AGU.

Chelton, D. B., R. A. De Szoeke, and M.G. Schlax (1998) Geographical variability of the first baroclinic Rossby radius of deformation. J Phys Oceanogr 28:433-460. doi: 10.1175/15200485(1998)028<0433:GVOTFB>2.0.CO;2.

Cheney, R. E., J. G. Marsh, and B. D. Beckley (1983) Global mesoscale variability from collinear tracks of SEASAT altimeter data. J Geophys Res 88:4343-4354. doi: 10.1029/JC088iC07p04343.

Ducet, N., P. Y. Le Traon, and G. Reverdin (2000) Global high-resolution mapping of ocean circulation from TOPEX/Poseidon and ERS-1 and -2. J Geophys Res 105:19,477-19,498. doi: 10.1029/2000JC900063.

Feng, Y., W. Wang, and R. X. Huang (2006) Mesoscale available gravitational potential energy in the world oceans. Acta Oceanologica Sinica 25:1-13.

Ferrari, R., and C. Wunsch (2009) Ocean Circulation Kinetic Energy: Reservoirs, Sources, and Sinks. Annual Review of Fluid Mechanics 41:253-282. doi: 10.1146/annurev.fluid.40.111406.102139.

Ferrari, R., and C. Wunsch (2010) The distribution of eddy kinetic and potential energy in global ocean. Tellus A 62:92-108. doi:10.1111/j.1600-0870.2009.00432.x.

Flierl, G. R. (1978) Models of vertical structure and calibration of 2-layer models. Dynamics of Atmospheres and Oceans 2:341-381. doi:10.1016/0377-0265(78)90002-7.

Forget, G., and C. Wunsch (2007) Estimated global hydrographic variability. J Phys Oceanogr 37:1997-2008. doi:10.1175/JPO03072.1.

Frankignoul, C., and P. Muller (1979) Quasi-geostrophic response of an infinite beta-plane ocean to stochastic forcing by the atmosphere. J Phys Oceanogr 9:104-127. doi:10.1175/15200485(1979)009<0104:QGROAI>2.0.CO;2.

$\mathrm{Fu}$, L., T. Keffer, P. Niiler, and C. Wunsch (1982) Observations of mesoscale variability in the western North Atlantic: A comparative study. J Mar Res 40:809-848.

Gill, A. E., Green, J. S. A. and Simmons, A. J. (1974) Energy partition in the large-scale ocean circulation and the production of mid-ocean eddies. Deep Sea Res 21:499-528. doi: 10.1016/00117471(74)90010-2.

Gille, S., M. Yale, and D. Sandwell (2000) Global correlation of mesoscale ocean variability with seafloor roughness from satellite altimetry. Geophys Res Lett 27:1251-1254. doi: 10.1029/1999GL007003.

39 Gould, W. J., W.J. Schmitz Jr., and C. Wunsch (1974) Preliminary field results for a Mid-Ocean 40 Dynamics Experiment (MODE-0). Deep Sea Res 21:911-931. doi: 10.1016/0011-7471(74)90025414.

42 Huang, R. X. (2005) Available potential energy in the world's oceans. J Mar Res 63:141-158. 43 doi:10.1357/0022240053693700.

44 Huang, R. X. (2010) Ocean circulation, wind-driven and thermohaline processes. Cambridge 45 University Press, Cambridge, United Kingdom, 806 pp.

46 Huang, R. X., and J. Pedlosky (2002) On aliasing Rossby waves induced by asynchronous time 47 stepping. Ocean Modelling 5:65-76. doi: 10.1016/S1463-5003(02)00014-8.

48 Huang, R. X., and W. Wang (2003) Gravitational potential energy sinks in the oceans, Near49 boundary processes and their parameterization. Proceedings, Hawaii winter workshop, pp239-247.

50 Killworth, P. D., and J. R. Blundell (2007) Planetary wave response to surface forcing and 51 instability in the presence of mean flow and topography. J Phys Oceanogr 37:1297-1320.

Lapeyre. G. (2009) What vertical mode does the altimeter reflect? On the decomposition in baroclinic modes and on a surface-trapped mode. J Phys Oceanogr 39:2857-2874. doi: 10.1175/2009JPO3968.1.

55 Oort, A. H., L. A. Anderson, and J. P. Peisxoto (1994) Estimates of the energy cycle of the oceans.

56 J Geophys Res 99:7665-7688. doi: 10.1029/93JC03556.

57 Pedlosky, J. (1987) Geophysical Fluid Dynamics. Springer-Verlag, New Work, 710 pp.

58 Richardson, P. L. (1983) Eddy kinetic energy in the North Atlantic from surface drifters. J 59 Geophys Res 88:4355-4367. doi: 10.1029/JC088iC07p04355.

60 Roemmich, D., and J. Gilson (2001) Eddy transport of heat and thermo-cline waters in the North

61 Pacific: A key to interannual/decadal climate variability? J Phys Oceanogr 31:675-687.

62 doi:10.1175/1520-0485(2001)031<0675:ETOHAT>2.0.CO;2 
Scott, R., and Y. Xu (2009) An update on the wind power input to the surface geostrophic flow of the World Ocean. Deep Sea Res Part I 56:295-304. doi: 10.1016/j.dsr.2008.09.010.

Sen, A., R. Scott, and B. Arbic (2008) Global energy dissipation rate of deep-ocean low-frequency flows by quadratic bottom boundary layer drag: Computations from current-meter data. Geophys Res Lett 35:L09606. doi: 10.1029/2008GL033407.

Shum, C. K., R. A. Werner, D. T. Sandwell, B. H. Zhang, R. S. Nerem, and B. D. Tapley (1990) Variations of global mesoscale eddy energy observed from GEOSAT. J Geophys Res 95:1786517876. doi: 10.1029/JC095iC10p17865.

Smith, K. (2007) The geography of linear baroclinic instability in Earth's oceans. J Mar Res 65:655-683. doi: 10.1357/002224007783649484.

Stammer, D. (1997) Global characteristics of ocean variability estimated from regional TOPEX/POSEIDON altimeter measurements. J Phys Oceanogr 27:1743-1769. doi: 10.1175/15200485(1997)027<1743:GCOOVE>2.0.CO;2.

Stammer, D., C. B ning, and C. Dieterich (2001) The role of variable wind forcing in generating eddy energy in the North Atlantic. Progress in Oceanography 48:289-311. doi: 10.1016/S00796611(01)00008-8.

Wang, G., J. Su, and P. Chu (2003) Mesoscale eddies in the South China Sea observed with altimeter data. Geophys Res Lett 30:2121. doi: 10.1029/2003GL018532.

Wunsch, C. (1997) The vertical partition of oceanic horizontal kinetic energy. J Phys Oceanogr 27:1770-1794. doi: 10.1175/1520-0485(1997)027<1770:TVPOOH>2.0.CO;2.

Wunsch, C. (1998) The work done by the wind on the oceanic general circulation. J Phys Oceanogr 28:2332-2340. doi: 10.1175/1520-0485(1998)028<2332:TWDBTW>2.0.CO;2.

Wunsch, C. (2007) The past and future ocean circulation from a contemporary perspective, Ocean circulation: mechanisms and impacts: past and future changes of meridional overturning, No. 173, Geophysical Monograph-American Geophysics Union, 53-74.

Wunsch, C., and R. Ferrari (2004) Vertical mixing, energy and the general circulation of the oceans. Ann Rev Fluid Mech 36:281-314. doi: 10.1146/annurev.fluid.36.050802.122121.

Wyrtki, K., L. Magaard, and J. Hager (1976) Eddy Energy in the Oceans. J Geophys Res 81:26412646. doi: 10.1029/JC081i015p02641.

Yuan, D., W. Han, and D. Hu (2006) Surface Kuroshio path in the Luzon Strait area derived from satellite remote sensing data. J Geophys Res 111:C11007. doi: 10.1029/2005JC003412.

Zamudio, L., H. E. Hurlburt, E. J. Metzger, and C. E. Tilburg (2007) Tropical wave-induced oceanic eddies at Cabo Corrientes and the María Islands, Mexico. J Geophys Res 112:C05048. doi: 10.1029/2006JC004018.

35 Zlotnicki, V., L. L. Fu, and W. Patzert (1989) Seasonal Variability in Global Sea Level Observed 36 With Geosat Altimetry. J Geophys Res 94:17959-17969. doi: 10.1029/JC094iC12p17959. 
A first-baroclinic-mode eddy can be examined in terms of a two-layer model,

3 Fig. A1, where $\eta$ is the sea level anomaly, $h_{l}$ is the depth of the interface, $d$ is the

4 interfacial disturbance, $\left(H_{1}, H_{2}\right),\left(u_{1}, u_{2}\right),\left(\rho_{1}, \rho_{2}\right)$ are the mean thickness,

5 horizontal velocity and density of the upper and lower layers. The corresponding

6 pressure gradient in each layer is$$
\nabla p_{1}=g \rho_{1} \nabla \eta, \nabla p_{2}=g \rho_{1} \nabla \eta-g \Delta \rho \nabla h_{1}=g \rho_{1} \nabla \eta-g \Delta \rho \nabla d
$$

8 where $g$ is the gravitational acceleration, $\Delta \rho=\rho_{2}-\rho_{1}$ is the density difference

9 between the upper and lower layers. These relations can be rewritten as

$10 \quad \frac{\nabla p_{1}}{\rho_{1}}=g \nabla \eta, \frac{\nabla p_{2}}{\rho_{2}} \simeq g \nabla \eta-g^{\prime} \nabla d$,

11 where $g^{\prime}=g \Delta \rho / \rho_{2}$ is the reduced gravity. Geostrophic velocity in each layer is

12 proportional to the pressure gradient, the right panel of Fig. A1. By definition,

13 volumetric transport in each layer satisfy the following constraint

$14 \quad u_{1} H_{1}+u_{2} H_{2}=0$.

15 From these equations we obtain

$16 \quad d=\frac{g}{g^{\prime}}\left(1+\frac{H_{1}}{H_{2}}\right) \eta$.

17 Thus, the horizontal pressure term in the lower layer is reduced to

$18 \frac{\nabla p_{2}}{\rho_{2}} \simeq-g \frac{H_{1}}{H_{2}} \nabla \eta=-\frac{H_{1}}{H_{2}} \frac{\nabla p_{1}}{\rho_{1}}$.

19 When the lower layer is much thicker than the upper layer, velocity in the lower

20 layer is much smaller than that of the upper layer; however, the volumetric

21 transport in the lower layer is not negligible because it is exactly the same as that 
1 in the upper layer (with an opposite sign).

In the present two-layer model, if the lower layer is much thicker than the

3 upper layer, the layer ratio term in Eq. (4) can be omitted, and the corresponding

4 expression is reduced to

$5 \quad d \approx \frac{g}{g^{\prime}} \eta$.

6 However, in our calculation, the exact expression (4) for our 2-layer

7 approximation of the stratification is used.

8 The AGPE for a two-layer model can be calculated as follows. Assume the

9 undisturbed upper layer thickness is $H_{1}$, the free surface elevation is $\eta$ and the

10 interface depression is $d$, Fig. A2. The reference state is defined as the state with

11 minimal gravitational potential energy, which corresponds to a state with both the

12 free surface and the interfacial surface leveled off, as shown by the dashed

13 horizontal lines in Fig. A2. Since the vertical movement involved is very small,

14 we assume that water density does not change with pressure. As a result, the only

15 changes are as follows. First, the free surface elevation anomaly is flatted out, as

16 shown by the arrow in the upper part of Fig. A2. Second, the interface is flatted

17 out, indicated by the solid arrow in the lower part of Fig. A2. However, other parts

18 of upper and lower layer remain unchanged.

19 The calculation of AGPE is separated into two parts. For the upper part of the

20 water column, we use the upper surface of the undisturbed upper layer as the

21 reference state. The total gravitational potential energy of the water parcel before

22 and after adjustment is

$23 \chi_{\text {Top }}^{0}=\frac{g \rho_{1} b}{2} \eta^{2}$,

$24 \quad \chi_{\text {Top }}^{1}=\frac{g \rho_{1}}{2}(B+b) a^{2}=\frac{g \rho_{1}}{2} \frac{b^{2}}{B+b} \eta^{2}$. 
1 Thus, the corresponding available gravitational potential energy is

$2 \Delta \chi_{\text {Top }}=\chi_{\text {Top }}^{0}-\chi_{\text {Top }}^{1}=\frac{g \rho_{1}}{2} \frac{B b}{B+b} \eta^{2}$.

3 For the lower part of the water column near the interface, there are two water

4 parcels exchanging their positions. For simplicity, we use the non-disturbed

5 interface as the reference level. Before the adjustment, the total gravitational

6 potential energy for the upper layer parcel (on the lower-left corner) and the lower

7 layer (on the lower-right corner) is

$8 \quad \chi_{B o t, 1}^{0}=-\frac{g \rho_{1}}{2} b(d-e)^{2}$,

$9 \quad \chi_{B o t, 2}^{0}=\frac{g \rho_{2}}{2} B e^{2}$.

10 The corresponding terms after adjustment have similar expressions,

$11 \chi_{B o t, 1}^{1}=\frac{g \rho_{1}}{2} B e^{2}$,

$12 \chi_{B o t, 2}^{1}=-\frac{g \rho_{2}}{2} b(d-e)^{2}$.

13 Thus, the available gravitational potential energy associated with the adjustment

14 of these two water parcels are

$15 \Delta \chi_{B o t}=\chi_{B o t, 1}^{0}+\chi_{B o t, 2}^{0}-\chi_{B o t, 1}^{1}-\chi_{B o t, 2}^{1}=\frac{g \Delta \rho}{2} \frac{b B}{(B+b)} d^{2}$.

16 For an individual eddy, the width of the background stratification field is

17 much larger than the width of the eddy, so that $B \gg b$, and the corresponding total

18 available gravitational potential energy for the unit length, obtained by dividing

19 the width of $b$, is

$20 \quad \chi=\Delta \chi_{\text {Top }}+\Delta \chi_{\text {Bot }} \approx \frac{g \Delta \rho}{2} d^{2}+\frac{g \rho_{1}}{2} \eta^{2}$.

21 Using Eq. (4), this is reduced to 


$$
1 \quad \chi \simeq \frac{g \Delta \rho}{2}\left[1+\frac{g^{\prime}}{g}\left(\frac{H_{2}}{H_{1}+H_{2}}\right)^{2}\right] d^{2} .
$$

2 Since the reduced gravity is much smaller than gravity, the second term in Eq.

3 (A14) is negligible and the total available gravitational potential energy per unit

4 length is

$5 \quad \chi \simeq \frac{g \Delta \rho}{2} d^{2}$.

6 Our discussion above can be extended to the case of an eddy in a cylindrical

7 coordinates. Assuming eddy dimension is much smaller than the dimension of the

8 ocean, the results are the same.

9 The ratio of EAGPE and EKE for an eddy is estimated as follows. The 10 geostrophic velocity of an eddy in the upper layer is estimated as

$11 \quad u_{1}=g|\nabla \eta| / f \simeq g \eta_{\max } / f r$,

12 where $f=2 \Omega \sin \theta$ is the Coriolis parameter, $\Omega$ is the earth rotation rate, $\theta$ is the

13 latitude, $\eta_{\max }$ is the maximal free surface elevation at the center of the eddy and

$14 r$ is the radius of the eddy. Therefore, the total amount of kinetic energy of an

15 eddy integrated over the total area of the eddy, $A$, is estimated as

$16 \quad E_{k}=\frac{1}{2} \rho_{1} H_{1}\left(1+\frac{H_{1}}{H_{2}}\right) \iint_{A} u^{2} d A \simeq \frac{1}{2} \rho_{1} H_{1}\left(1+\frac{H_{1}}{H_{2}}\right)\left(\frac{g \eta_{\max }}{f r}\right)^{2} A$.

17 The corresponding total available gravitational potential energy of an eddy is

18 estimated as

$19 \quad E_{\text {agpe }} \simeq \frac{1}{2} \Delta \rho g\left[\frac{g}{g^{\prime}}\left(1+\frac{H_{1}}{H_{2}}\right) \eta_{\max }\right]^{2} A$.

20 Thus, the ratio of these two types of energy for an eddy is

$21 R=\frac{E_{\text {agpe }}}{E_{k}} \simeq\left(\frac{r}{r_{d}}\right)^{2}$, 
1 where $r_{d}=\sqrt{g^{\prime} H_{1} H_{2} /\left(H_{1}+H_{2}\right)} / f$ is the first radius of deformation. Thus, for

2 eddy with radius close to the first deformation radius, the total energy is roughly

3 equally partitioned between the EAGPE and EKE. However, most eddies

4 identifiable from the oceanic datasets, especially from the altimetry, the horizontal

5 length scale is much larger than the first radius of deformation (Chelton et al.

6 2007; Stammer, 1997; Roemmich and Gilson, 2001). As a result, the eddy energy

7 is mostly in the form of EAGPE.

8

9 Appendix B: 2.2. Inferring the two-layer model from a continuously stratified model

11 A vitally important step in formulating the two-layer model is to specify the equivalent depth of the mean interface and the density difference between the two

13 layers. A simple approach is to use the depth of the main pycnocline and the 14 associated density jump. In the following discussion this model will be called the

15 thermocline model (TH-model). Such a model is, however, not suitable for the

16 subpolar basin and the Southern Ocean where the main thermocline is poorly 17 defined.

18 A better approach in parameterization of a two-layer model was described by

19 Flierl (1978). Mesoscale eddy can be described in terms of the normal modes, and

20 the standard formulation has been described in many previous literatures, e.g.,

21 Pedlosky (1987), Chelton et al. (1998), and Huang and Pedlosky (2002). Our 22 notation here follows Flierl (1978). The normal modes can be defined as the 23 following eigen value/function problem:

$24 \frac{d}{d z}\left(\frac{f^{2}}{N^{2}} \frac{d F_{n}}{d z}\right)+\lambda_{n} F_{n}=0$, 
$1 \quad \frac{d F_{n}}{d z}=0, z=0,-H$

2 where $F_{n}(z)$ is the n-th eigen mode, $\lambda_{n}$ is the corresponding eigen value, $N^{2}$ is the

3 squared buoyancy frequency, and $H$ is the depth of the sea floor. A normalization

4 constraint is also applied to the eigen functions

$5 \quad \int_{-H}^{0} F_{i} F_{j} d z=H \delta_{i j}$.

6 Our study is focused on the first baroclinic mode. The choice of parameter

7 for a two-layer model depends on the physical aspects of the problem as discussed

8 by Flierl (1978). Unfortunately, no suitable formulation specifically designed for

9 the study of the available potential energy is available at present time; thus, we

10 will adapt the standard formulation for normal mode presented by Flierl (1978).

11 Accordingly, the equivalent interface depth and the equivalent density step are

$12 \quad H_{1}=\frac{H}{1+F_{1}^{2}(0)}$,

$13 \varepsilon=\frac{f_{0}^{2} H}{\lambda_{1} g H_{1}\left(H-H_{1}\right)}$.

14 The equivalent reduced gravity is defined as

$15 \quad g^{\prime}=\varepsilon g$.

16 This model will be called the equivalent two-layer model (EQ-model).

Appendix C: Calculation of the annual mean generation/dissipation rate of mesoscale eddies

20 Through eddy identification and tracking, the time series of position and

21 energy for an eddy were obtained and the total energy of an eddy at each moment

22 during its lifetime were calculated as summation of EKE and EAGPE. The

23 detailed algorithm of annual mean generation and dissipation rate of the 
1 mesoscale eddy is as follows.

Assume that we have a time series of an eddy, including its position and the

3 SSHA at time $t=t_{1}, t_{2}, \ldots, t_{n-1}$ with uniform time step of one week. In order to

4 analyze the life cycle of an eddy, we need to define the beginning and end of the

5 eddy. The beginning of an eddy is with zero energy, so that $e_{0}=0$, and its time is

6 defined as $t_{0}=-2 t_{1}+t_{2}$; its position is defined by a linear extrapolation from

7 point 1 and 2: $\left(x_{0}, y_{0}\right)=\left(-2 x_{1}+x_{2},-2 y_{1}+y_{2}\right)$. Similarly, the end of the eddy can

8 be defined.

9 The energy source or sink within each pair of points $d e_{i, i+1}$ is calculated as

$10 d e_{01}=e_{1}-e_{0}=e_{1}$,

$11 d e_{i, i+1}=e_{i+1}-e_{i}$,

12 The location of $d e_{i, i+1}$ is in the middle of these two positions.

13 Gridded energy variation data set was required, so the $1^{\circ} \times 1^{\circ}$ grid was

14 chosen here. Suppose we have four grid points: $(i, j),(i+1, j),(i, j+1),(i+1, j+1)$, the

15 contributions to four grid points were calculated by the method of weighting. We

16 assume there is a point source de locates $(m, n)$ with a non-dimensional position

$17(X, Y)$ within this grid net, $X=m-i, Y=n-j$. Thus, contribution of this source to the

18 grid points at the four comers is:

$19 e(i, j)=d e(1-X)(1-Y)$,

$20 \quad e(i+1, j)=d e X(1-Y)$

$21 e(i, j+1)=d e(1-X) Y$,

$22 e(i+1, j+1)=d e X Y$.

23 As a result, in 15-year accumulation the total contribution of these sources or

24 sinks at those grid points is: 
$1 \quad E_{i, j}^{\text {source }}=\sum_{n=1, \text { for } e_{n}>0}^{N} e_{n}$,

$2 \quad E_{i, j}^{\mathrm{sink}}=\sum_{n=1, \text { for } e_{n}<0}^{N} e_{n}$.

3 The total contribution of these sources or sinks at each grid point divided by the

4 15-year time is the annual mean generation and dissipation rate of mesoscale

5 eddies:

$6 \quad w_{i, j}^{\text {source }}=E_{i, j}^{\text {source }} / T$,

$7 \quad w_{i, j}^{\mathrm{sink}}=E_{i, j}^{\mathrm{sink}} / T$.

Appendix D: Results in TH-model

10 Results from the TH-model are much smaller than the corresponding values

11 obtained from the EQ-model, and the global sum of eddy energy generation rate is

12 estimated at $0.113 \mathrm{TW}$ (Table 3). In particular, the contribution from the ACC in

13 the EQ-model is also much higher than that obtained from the TH-model. Such

14 difference is due to the fact that the TH-model underestimates both the depth of

15 the equivalent interface and the density jump across the interface, as shown in

$16 \quad$ Figs. 2 and 3.

17 Accordingly, for the TH-model the EKE generation rate of cyclonic eddies is

18 slightly lower than that of anticyclonic eddies. However, the EAGPE generation

19 rate of cyclonic eddies is slightly higher than that of anticyclonic eddies. For the

20 global sums, in the TH-model the EAGPE generation rate is 1.15 times larger than

21 that of EKE (Table 4).

22 Since the interface depth in the TH-model is not suitable for the eddy in the 
1 subpolar basin and the Southern Ocean where the main thermocline is poorly

2 defined, we present the results from the TH-model as a comparison and a 3 sensitivity test. 

two-layer model, in EJ $\left(10^{18} \mathrm{~J}\right)$.

\begin{tabular}{lllll}
\hline $\begin{array}{l}\text { Resolution } \\
\text { used } \begin{array}{l}\text { in } \\
\text { smoothing }\end{array}\end{array}$ & Cyclonic eddies & $\begin{array}{l}\text { Anticyclonic } \\
\text { eddies }\end{array}$ & Sum \\
\hline \multirow{2}{*}{$6^{\circ} \times 6^{\circ}$} & EKE & 0.081 & 0.076 & 0.157 \\
\cline { 2 - 5 } & EAGPE & 0.113 & 0.111 & 0.224 \\
\cline { 2 - 5 } & Sum & 0.194 & 0.187 & 0.381 \\
\hline \multirow{2}{*}{$5^{\circ} \times 5^{\circ}$} & EKE & 0.044 & 0.041 & 0.085 \\
\cline { 2 - 5 } & EAGPE & 0.056 & 0.055 & 0.111 \\
\cline { 2 - 5 } & Sum & 0.100 & 0.096 & 0.196 \\
\hline \multirow{2}{*}{$7^{\circ} \times 7^{\circ}$} & EKE & 0.119 & 0.113 & 0.232 \\
\cline { 2 - 5 } & EAGPE & 0.174 & 0.171 & 0.345 \\
\cline { 2 - 5 } & Sum & 0.293 & 0.284 & 0.577 \\
\hline
\end{tabular}

Table 2. Global sum of EKE and AGPE, in EJ $\left(10^{18} \mathrm{~J}\right)$

\begin{tabular}{|c|c|c|c|c|c|c|}
\hline & \multirow{2}{*}{$\begin{array}{l}\text { Equivalent } \\
\text { 2-layer } \\
\text { model }\end{array}$} & \multicolumn{2}{|c|}{ Feng et al. (2006) } & \multirow{2}{*}{$\begin{array}{l}\text { Huang } \\
\text { (2005) }\end{array}$} & \multirow{2}{*}{$\begin{array}{l}\text { Huang } \\
(2010)\end{array}$} & \multirow{2}{*}{$\begin{array}{l}\text { Ferrari \& } \\
\text { Wunsch } \\
\text { (2009) }\end{array}$} \\
\hline & & $1^{\circ} \times 1^{\circ}$ grid & $2^{\circ} \times 2^{\circ}$ grid & & & \\
\hline AGPE & 0.224 & 1 & 8 & $1880(810)$ & & \\
\hline EKE & 0.157 & & & & 1.46 & 2.6 \\
\hline
\end{tabular}

6 Table 3. Total generation/dissipation rate for eddies with lifetime $\geq 2$ weeks, in GW.

\begin{tabular}{|c|c|c|c|c|c|c|}
\hline $\begin{array}{l}\text { Resolution } \\
\text { used in } \\
\text { smoothing }\end{array}$ & & Eddy types & $\mathrm{NH}$ & $\mathrm{SH}$ & $\mathrm{ACC}$ & Global \\
\hline \multirow{3}{*}{$6^{\circ} \times 6^{\circ}$} & \multirow{3}{*}{$\begin{array}{c}\text { Equivalent } \\
\text { two-layer } \\
\text { model }\end{array}$} & Cyclonic & 32 & 71 & $49.7(48.3 \%)$ & 103 \\
\hline & & Anticyclonic & 32 & 68 & $47.4(47.4 \%)$ & 100 \\
\hline & & Sum & 64 & 139 & $97.1(47.8 \%)$ & 203 \\
\hline \multirow{3}{*}{$6^{\circ} \times 6^{\circ}$} & \multirow{3}{*}{$\begin{array}{l}\text { Thermocline } \\
\text { model }\end{array}$} & Cyclonic & 29 & 29 & $12.0(20.7 \%)$ & 58 \\
\hline & & Anticyclonic & 28 & 27 & $11.0(20.0 \%)$ & 55 \\
\hline & & Sum & 57 & 56 & $23.0(20.3 \%)$ & 113 \\
\hline \multirow{3}{*}{$5^{\circ} \times 5^{\circ}$} & \multirow{3}{*}{$\begin{array}{c}\text { Equivalent } \\
\text { two-layer } \\
\text { model }\end{array}$} & Cyclonic & 18 & 39 & $27.2(47.7 \%)$ & 57 \\
\hline & & Anticyclonic & 18 & 37 & $25.7(46.7 \%)$ & 55 \\
\hline & & Sum & 36 & 76 & $52.9(47.2 \%)$ & 112 \\
\hline \multirow{3}{*}{$7^{\circ} \times 7^{\circ}$} & \multirow{3}{*}{$\begin{array}{c}\text { Equivalent } \\
\text { two-layer } \\
\text { model }\end{array}$} & Cyclonic & 46 & 104 & $72.1(48.7 \%)$ & 150 \\
\hline & & Anticyclonic & 45 & 99 & $68.5(47.6 \%)$ & 144 \\
\hline & & Sum & 91 & 203 & $52.9(47.2 \%)$ & 294 \\
\hline
\end{tabular}

NH means the Northern Hemisphere, SH means the Southern Hemisphere (including the ACC band) and ACC means the zonal band from $40^{\circ} \mathrm{S}$ to $60^{\circ} \mathrm{S}$. The percentages indicate the proportions of the part energy conversion rates by the total.

11 Table 4. Global generation/dissipation rate for eddies with lifetimes $\geq 2$ weeks, in GW. model Equivalent 2-layer model $\quad \begin{gathered}\text { Thermocline } \\ \text { model }\end{gathered}$ 


\begin{tabular}{cccccc}
\hline \multicolumn{2}{c}{$\begin{array}{c}\text { Resolution used in } \\
\text { smoothing }\end{array}$} & $6^{\circ} \times 6^{\circ}$ & $5^{\circ} \times 5^{\circ}$ & $7^{\circ} \times 7^{\circ}$ & $6^{\circ} \times 6^{\circ}$ \\
\hline \multirow{3}{*}{ EKE } & Cyclonic & 45 & 26 & 64 & 27 \\
\cline { 2 - 6 } & Anticyclonic & 43 & 25 & 60 & 25 \\
\cline { 2 - 6 } & Total & 88 & 51 & 124 & 52 \\
\cline { 2 - 6 } EAGPE & Cyclonic & 58 & 31 & 86 & 30 \\
\cline { 2 - 6 } & Anticyclonic & 57 & 30 & 84 & 31 \\
\cline { 2 - 6 } & Total & 115 & 61 & 170 & 60 \\
\hline Total & & 203 & 112 & 294 & 113 \\
\hline
\end{tabular}


3 Fig. 1 (top) The trajectories of (a) cyclonic and (b) anticyclonic eddies with lifetimes $\geq 4$ weeks in 4 North Atlantic in 1993.

(middle) The number per $1^{\circ}$ square of long-lived eddies in 15 years, (c) is for cyclonic eddies and 6 (d) is for anticyclonic eddies. The interval between contours is 5.

7 (bottom) The global mean EKE (per unit mass) calculates as $0.5 \times\left(u^{2}+v^{2}\right)$ in unit of $\mathrm{cm}^{2} \mathrm{~s}^{-2}$, where $u$, $8 \mathrm{v}$ are zonal and meridional geostrophic velocities. (e) is plotted in $\log _{10}$ form while (f) is not.

Fig. 2 The global map of a) the equivalent interface depth $\mathrm{H}_{1}$ of the equivalent 2-layer model and b) the depth of main thermocline, in $\mathrm{m}$. The black heavy solid line indicates the $200 \mathrm{~m}$-isobath, which marks the boundary of data domain.

Fig. 3 The global map of a) the density step $\varepsilon=g^{\prime} / g$ derived from the Eq. (B4) and b) the density step $\varepsilon$ derived from the depth of main thermocline. It is dimensionless. The black solid line indicates the $200 \mathrm{~m}$-isobath.

Fig. 4 Meridional distribution of eddy properties, based on the equivalent two-layer model. a) Zonally integrated energy of cyclonic eddies. The solid line indicates the zonally integrated EKE while the dashed line indicates the zonally integrated EAGPE. b) Zonal mean deformation radius. c) Zonal mean lifetime of cyclonic eddies. d) The thick solid line indicates the zonal mean ratio of eddy radius over the deformation radius. The thin solid line indicates the zonal mean ratio of EAGPE/EKE while the thin dashed line indicates double the ratio of EAGPE/EKE. The dotted line indicates the ratios equal to 1 .

Fig. 5 The ratio of EAGPE/EKE whose resolution is $1^{\circ} \times 1^{\circ}$. The ratio larger than 5 is set to 5 . The black solid line indicates the 200m-isobath.

Fig. 6 The mean energy generation rate of cyclonic eddies with lifetimes $\geq 2$ weeks, in $\mathrm{mW} / \mathrm{m}^{2}$. The black solid line indicates the $200 \mathrm{~m}$-isobath.

Fig. 7 The zonal (meridional) integration of global annual mean generation rate is shown in top (bottom), in GW/degree. Solid line indicates the annual mean generation rate of cyclonic eddies, dashed line indicates the annual mean generation rate of anticyclonic eddies.

Fig. 8 Top panel: Mean generation rate for cyclonic eddies with lifetime $\geq 2$ weeks, in $\mathrm{mW} / \mathrm{m}^{2}$. Lower panel: The ratio of EAGPE generation rates for cyclonic eddies over its EKE generation rates. The white thin line indicates the contour that ratio equals 1.5 and the black solid line indicates the $200 \mathrm{~m}$-isobath.

Fig. A1 Sketch of the free surface and the layer interface in a two-layer model: Left panel: Free surface and interface of a two-layer model. Right Panel: Velocity pattern of the first baroclinic mode. The symbols are explained in the main text.

Fig. A2 Water parcel movement during the adjustment to a state of minimal gravitational potential energy. 

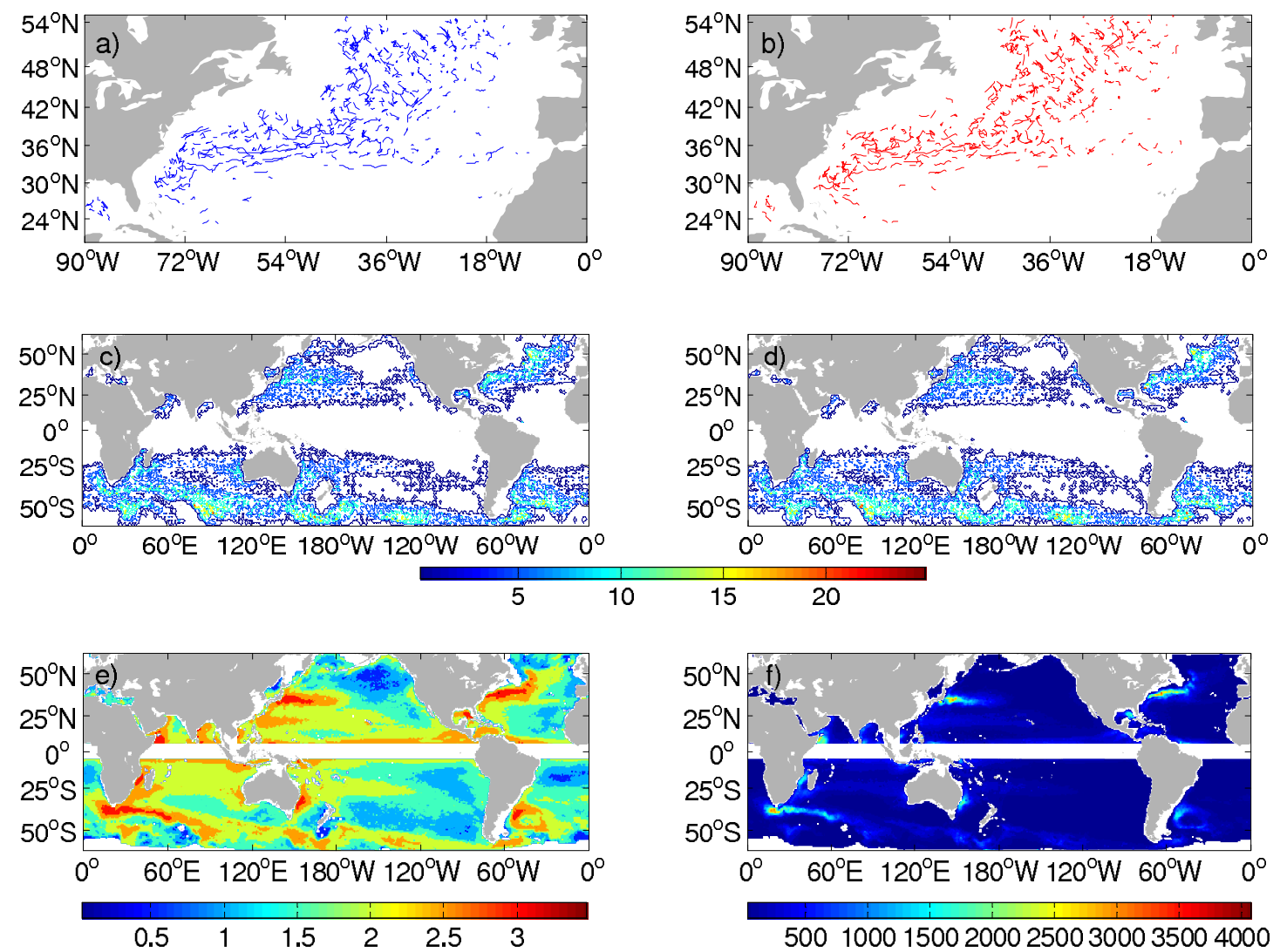

5001000150020002500300035004000

Fig. 1. Xu et al. 

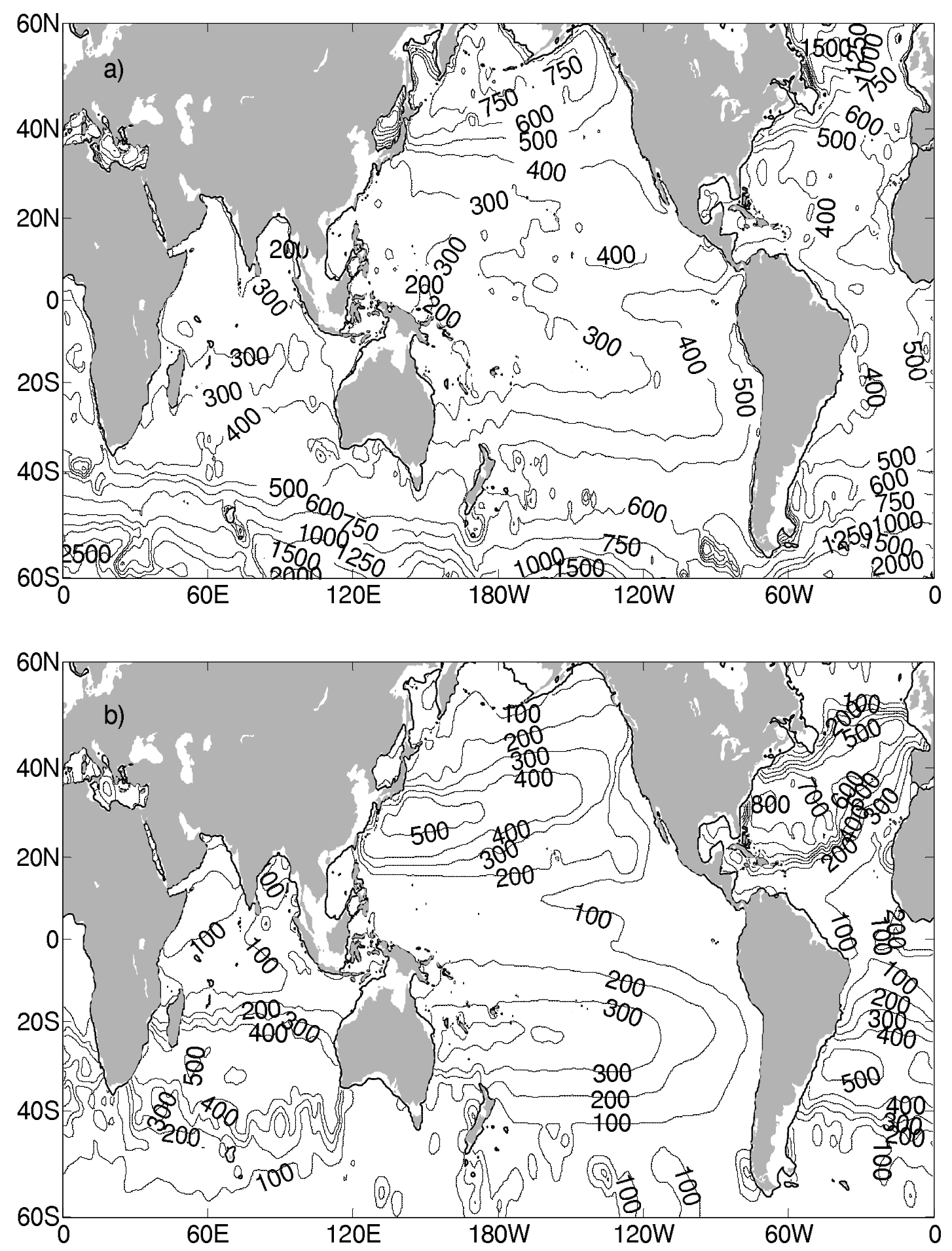

Fig. 2. Xu et al. 

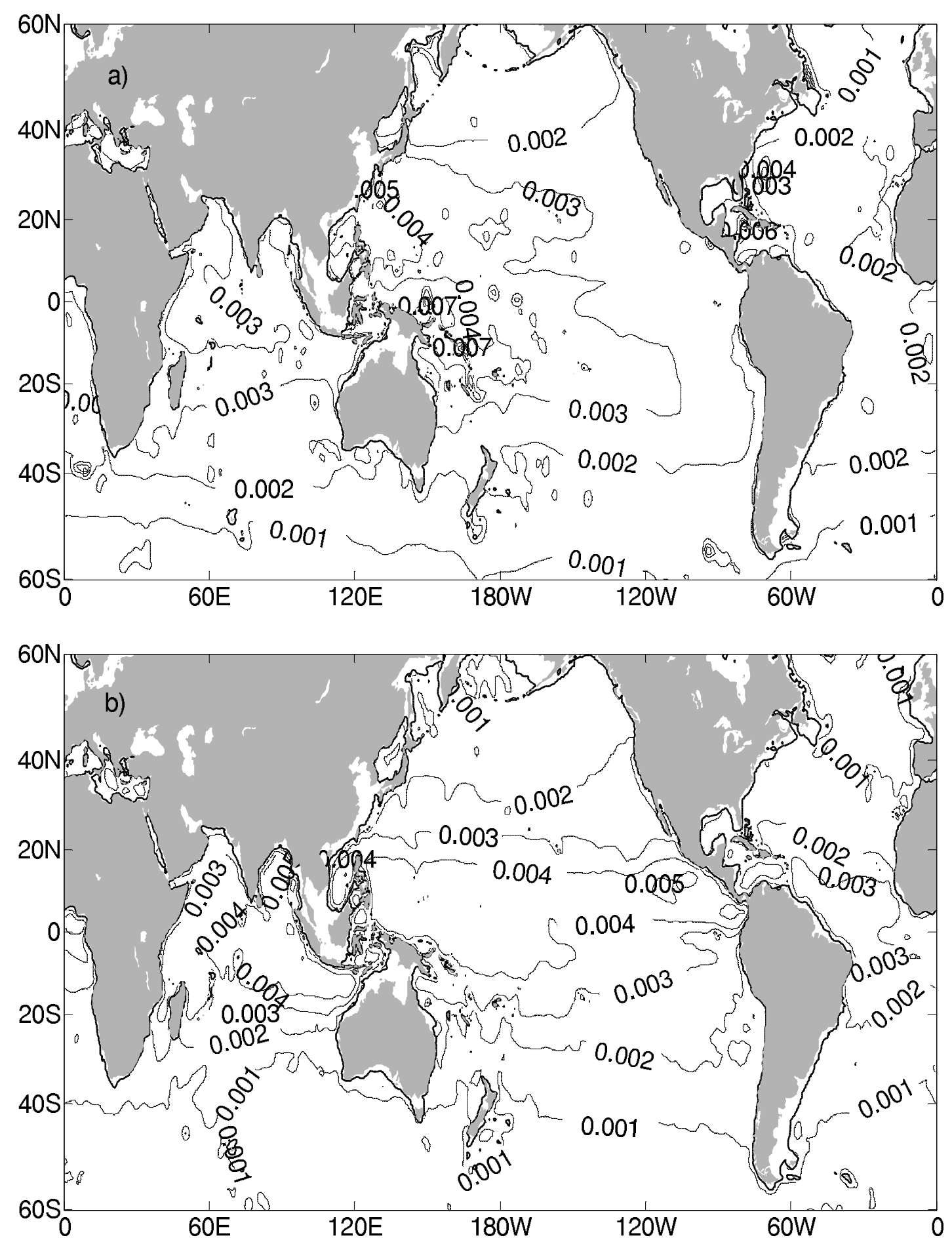

Fig. 3. Xu et al. 
a) Zonally integrated cyclonic eddy energy $\left(10^{15} \mathrm{~J}\right)$

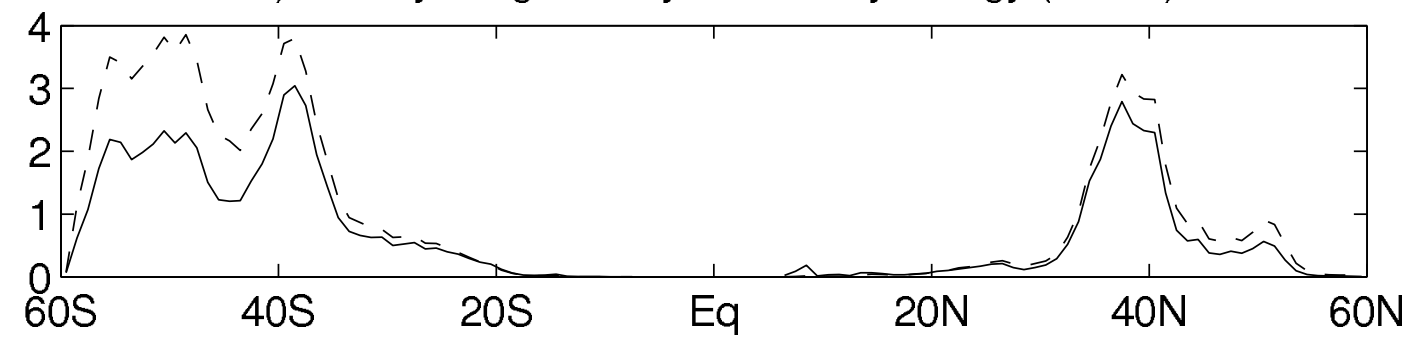

b) Zonal mean deformation radius $(\mathrm{km})$

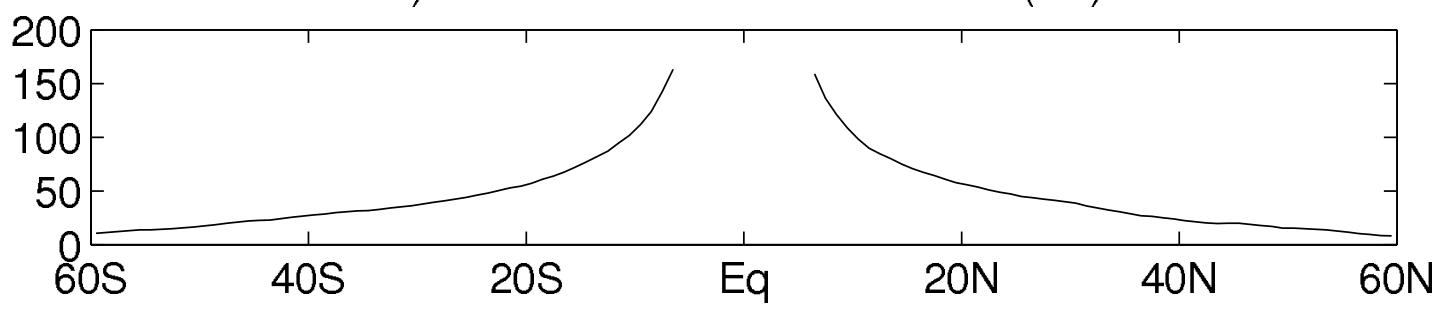

c) Zonal mean lifetime of cyclonic eddies (weeks)

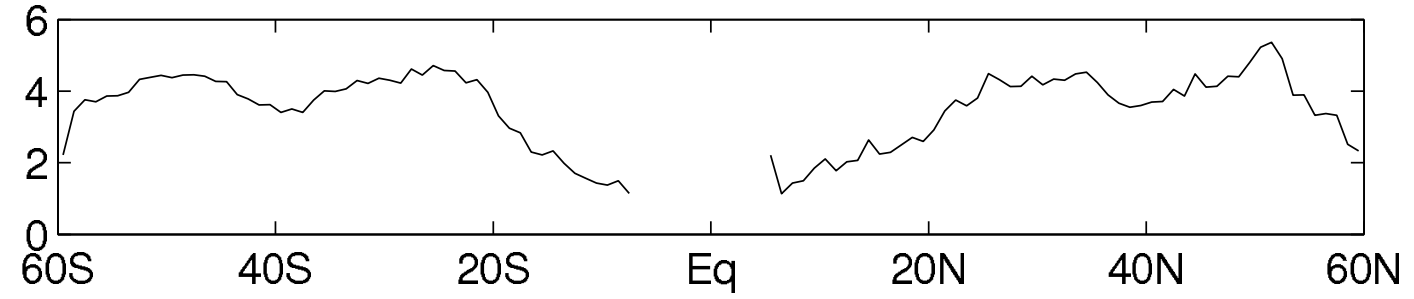

d) Zonal mean ratios

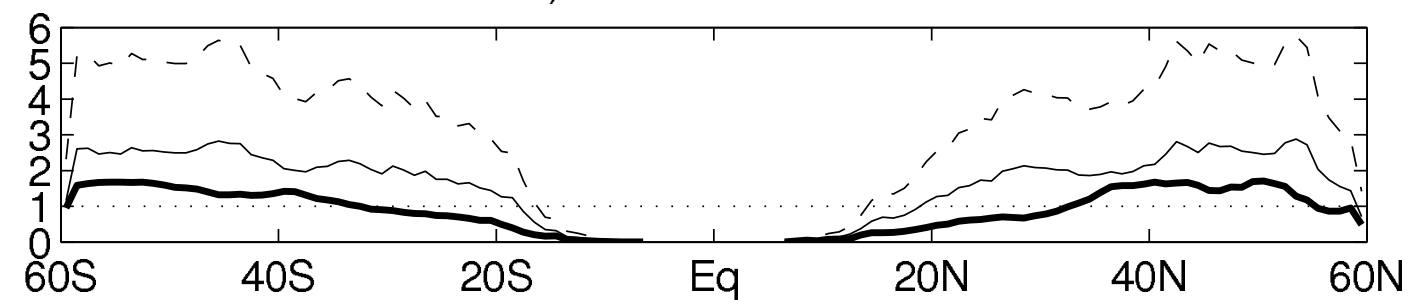

Fig. 4. Xu et al. 


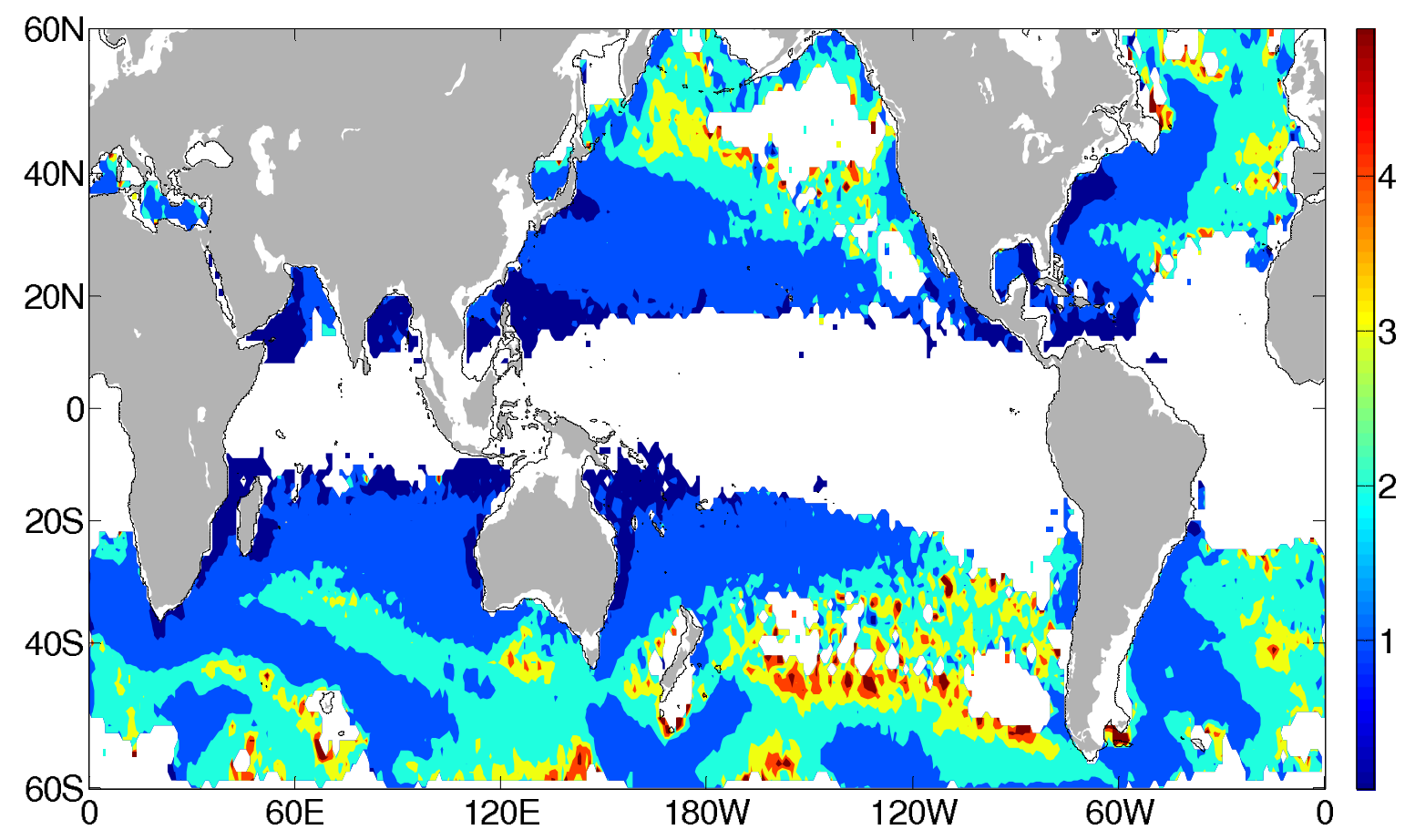

Fig. 5. Xu et al. 


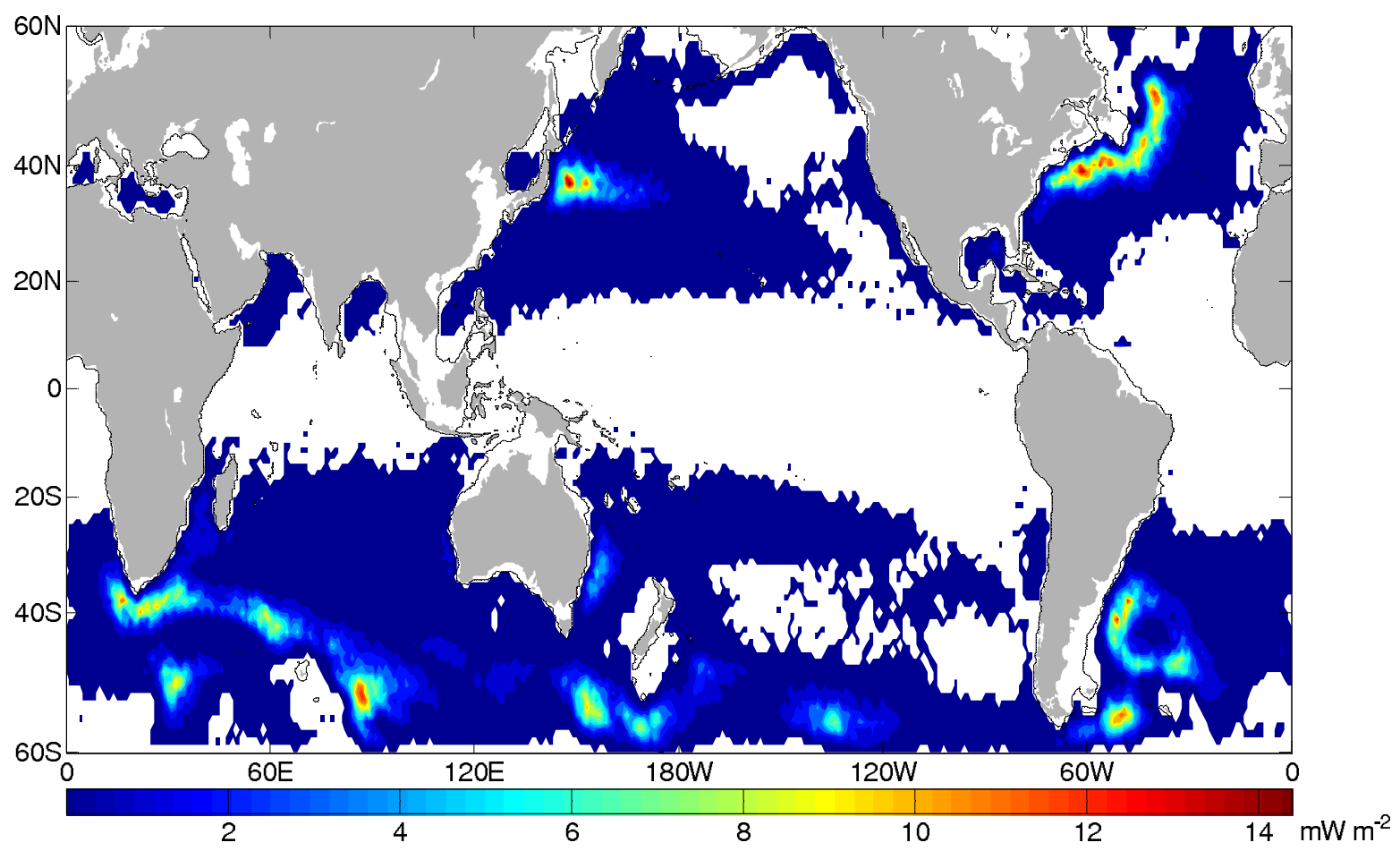

Fig. 6. Xu et al. 
a) Zonally integrated generation rate in GW

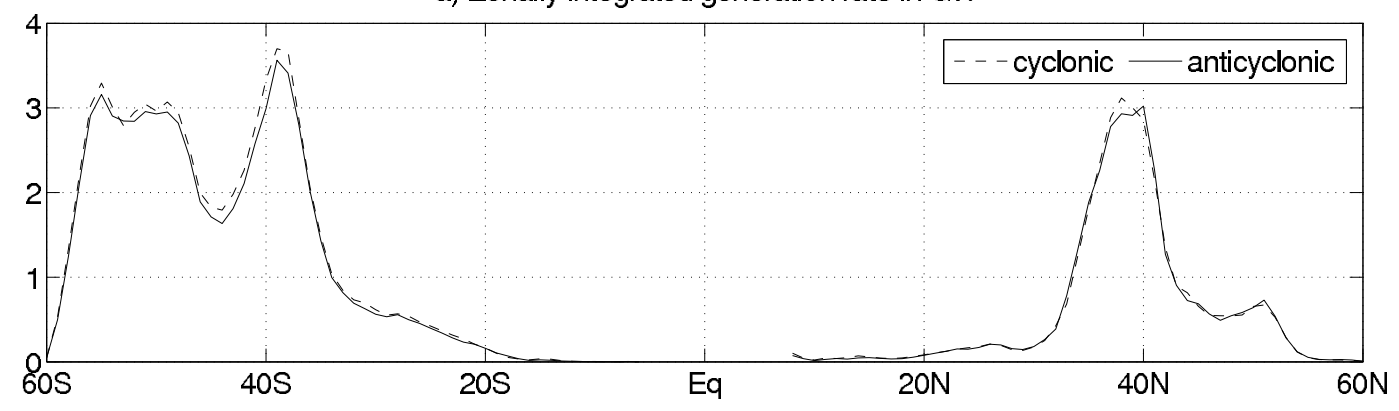

b) Meriodinal integrated generation rate in GW

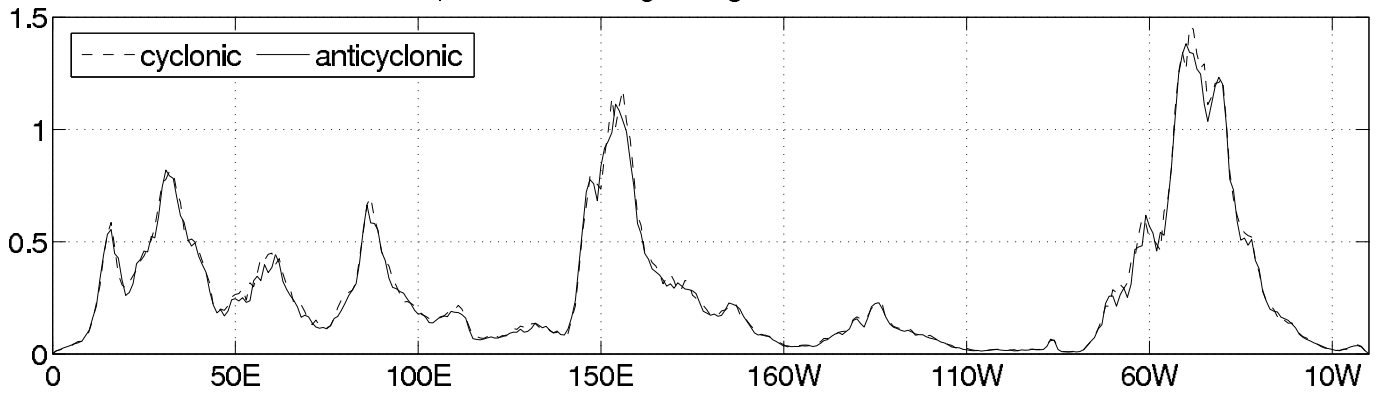

Fig. 7. Xu et al. 

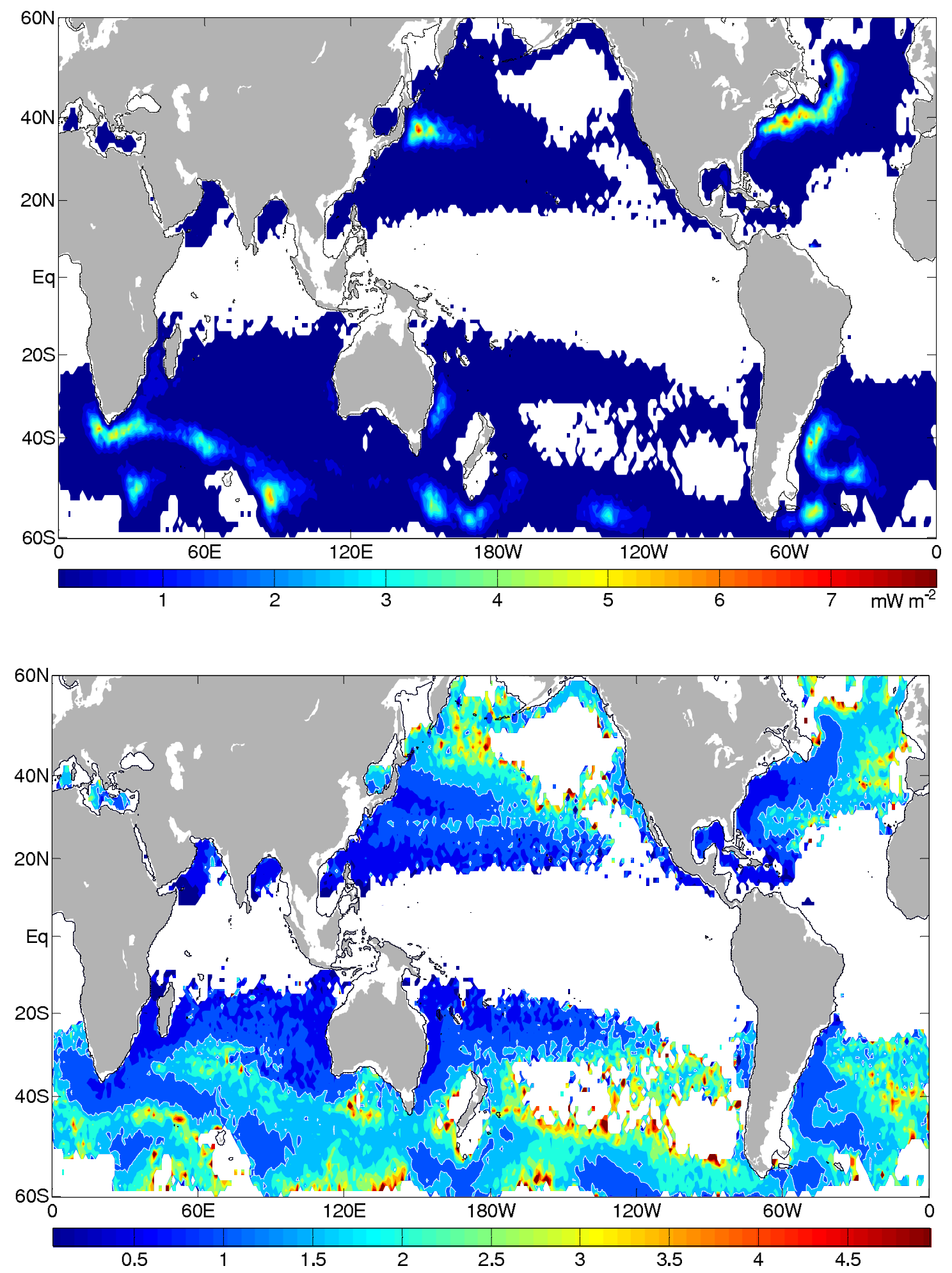

Fig. 8. Xu et al. 


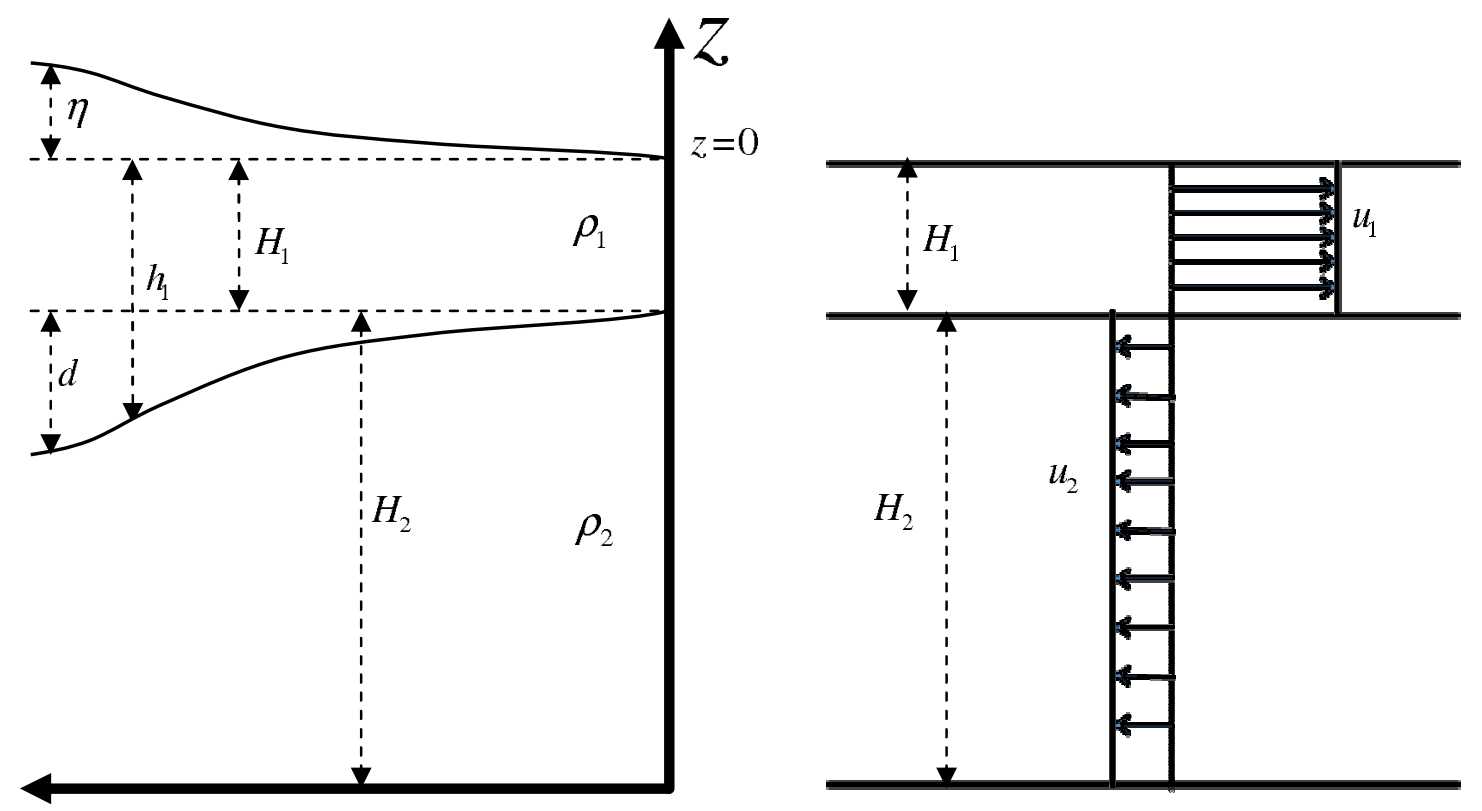

Fig. A1. Xu et al. 


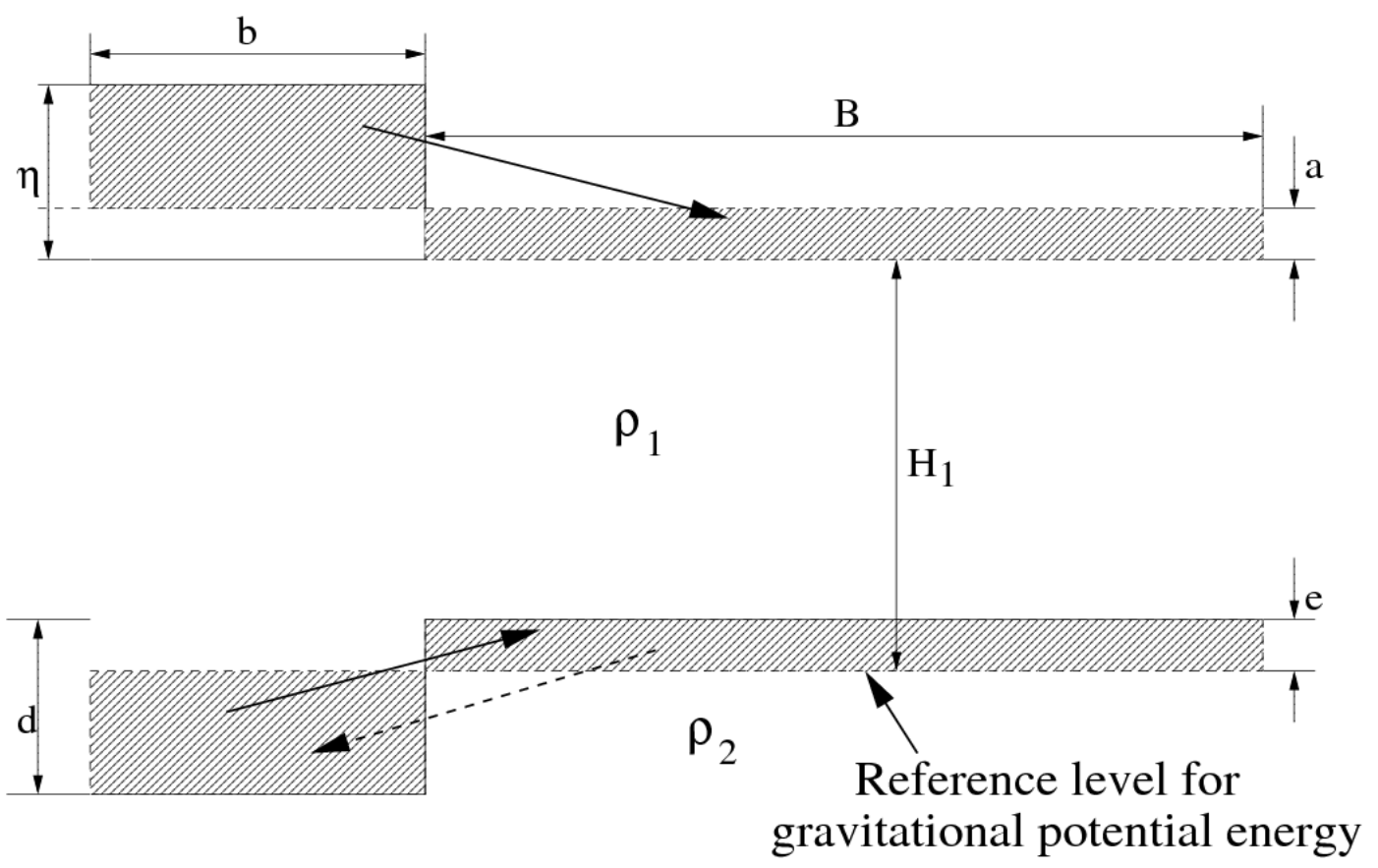

Fig. A2. Xu et al. 